\title{
Phase Transitions in One-Dimensional Nonlinear Viscoelasticity: Admissibility and Stability
}

\author{
RoBert L. Pego
}

Communicated by J. M. BALL

\begin{abstract}
For the motion of a one-dimensional viscoelastic material of rate type with a non-monotonic stress-strain relation, a mixed initial boundary value problem is considered. A simple existence theory is outlined, based on a novel transformation of the equation into the form of a degenerate reaction-diffusion system. This leads to new results characterizing the regularity of weak solutions. It is shown that each solution tends strongly to a stationary state asymptotically in time. Stable stationary states are characterized. Stable states may contain coexistent phases, i.e. they may have discontinuous strain. They need not be minimizers of energy in the strong sense of the calculus of variations; "metastable" and "absolutely stable" phases may coexist in a stable state. Furthermore, such states do arise as long-time limits of smooth solutions.

Beyond the above, "hysteresis" and "creep" phenomena are exhibited in a model of a loaded viscoelastic bar. Also, a viscosity criterion is proposed for the admissibility of propagating waves in the associated purely elastic model. This criterion is then applied to describe the formation of some propagating phase boundaries in a loaded elastic bar.
\end{abstract}

\section{Contents}

Part I: Approach to Equilibrium and Stability of Coexistent Phases in a Viscoelastic Bar . . . . . . . . . . . . . . . . . . . . . . . . . . 354

1. Introduction . . . . . . . . . . . . . . . . . . 354

2. Problem Description and Discussion . . . . . . . . . . . . . . 355

3. Local Existence and Regularity . . . . . . . . . . . . . . . . . . . 361

4. Exponentially Stable States . . . . . . . . . . . . . . . . . . . . . 367

5. Global Existence and Convergence to Equilibrium . . . . . . . . . . . . 373

6. Discontinuous Asymptotic States for Smooth Solutions . . . . . . . . . . 377

7. Hysteresis and Creep in a Load-Deformation Experiment . . . . . . . . . . 381 
Part II: Admissibility and Moving Phase Boundaries in an Elastic Bar . . . . 386

8. A Viscosity Criterion for Admissibility of Waves . . . . . . . . . . 386

9. Appearance of Propagating Phase Boundaries . . . . . . . . . . . . . . 389

Appendix: Some Estimates for the Abstract Parabolic Equation . . . . . . . . 391

References . . . . . . . . . . . . . . . . . . 393

\section{Part I. Approach to Equilibrium and Stability of Coexistent Phases in a Viscoelastic Bar}

\section{Introduction}

This article deals with a number of problems concerning a model equation for one-dimensional motion of a viscoelastic bar which may undergo phase changes. If $u(x, t)$ denotes the displacement at time $t$ of a reference point $x$ on the bar, the equation is

$$
u_{t t}=\left(\sigma\left(u_{x}\right)+\mu u_{x t}\right)_{x}, \quad \mu>0 .
$$

The stress $\sigma(w)$ is taken to be non-monotonic, for example of the form indicated in Fig. 1. Phases of the bar correspond to maximal intervals of monotonicity of $\sigma(w)$. The model is directly related to equations for isothermal motions of a van der Waals gas and equations for shearing motions in polymeric fluid ( $c f$. HUNTER \& SLemROD, 1983). It is allied with a model of a purely elastic bar proposed by ERICKSEN (1975), and has been studied by DAFERMOs (1969), ANDREWS (1980), and ANDrews \& BALL (1982).

The bulk of Part I below concerns issues of asymptotic behavior and the stability of states containing a mixture of phases, in a particular initial boundary value problem for (1.1) (see (2.3)). New results include: a simplified existence theory based on a transformation of problem (2.3) to one involving a semilinear system coupling a parabolic partial differential equation to an ordinary differential equation (see (2.9)); regularity results which establish that the strain $u_{x}$ must remain discontinuous if it is initially so, but that if the equaion is considered in conservation form, it is satisfied in an "almost classical" sense for $t>0$ (see (3.2)) ; strong convergence of solutions to a stationary state as $t \rightarrow \infty$; and identification of an uncountable infinity of dynamically stable stationary states (see (2.4), § 4 and $\S 6)$. The stability results establish that stationary states having discontinuous strain $u_{x}$ do arise as time-asymptotic limits of solutions with smooth strain.

The determination of dynamically stable stationary states given here is somewhat at odds with traditional stability analyses of phase equilibria. Originating with GiBBs (1906), such analyses identify stable states as states of minimum energy at constant entropy. Because dynamic processes dissipate energy, one usually expects the system to reach such a state. Multiple-phase states found stable dynamically for (2.3) below need not, however, be Gibbsian equilibria, states which minimize energy absolutely, nor be strong local minimizers. Instead, such states do minimize energy in a local sense, the weak sense of the calculus of variations; $c f . \S 2$ below. 
The possibilities indicated here may have physical interest in the study of phase changes in metals and other condensed media. Energy minimization arguments typically predict that phases can coexist only if the energy density in each phase is the same, at the absolute minimum. But, for example, austenitic and martensitic phases coexist in many metals in broad ranges of temperature ( $c f$. MülLER \& WILMANSKI, 1981). It is considered most unlikely that these phases maintain identical energy densities other than at isolated values of temperature. As a second example, twinned martensite is composed of symmetry-related phases, with equal energy densities at zero stress. When placed under a load which favors one twin, almost certainly the energy densities of the phases differ, yet they are observed to coexist. GIBBs himself recognized that "obstacles" may hinder a system from achieving a state of minimum energy. A deeper understanding of dynamic processes in the approach to equilibrium is called for when such obstacles are significant.

The remainder of this study addresses some related issues for dynamics involving phase mixtures in the model. For the viscoelastic model (1.1), hysteresis in stress vs. strain is exhibited in a dynamic loading-unloading process, not neglecting any inertial effects. A "creep" phenomenon is modeled at constant load. The description of these phenomena relies on the stability analysis of $\S 6$. Part II explores some consequences of taking the limit of vanishing viscosity in (1.1). A simple admissibility criterion for propagating waves in the associated purely elastic model is derived on the basis of this limit. Also an accountis proposed for the appearance of moving phase boundaries in a bar under an increased load, consistent with the admissibility criterion discussed. In experiments on bars under an increasing end load, the appearance of new phases can be associated with sharply defined, slowly moving waves. JAMEs (1980 b) describes some experimental literature on phase changes in polymers and metal bars.

\section{Problem Description and Discussion}

ERICKSEN (1975) suggested that the phenomena of phase transitions in bars might be modeled by the equation of one-dimensional elasticity,

$$
u_{t t}=\sigma\left(u_{x}\right)_{x},
$$

with nonmonotone $\sigma$. At rest, $\sigma\left(u_{x}\right)=P$ constant, so that several stationary states exist at a given load $P$, including states with coexistent phases, having discontinuous, piecewise constant strain $u_{x}$. For strain in ranges where $\sigma$ is decreasing, equation (2.1) is elliptic, making the initial value problem ill-posed. There is some hope that if strain is restricted to lie outside the elliptic range, the initial value problem might be rendered well-posed for weak solutions of the associated system,

$$
\begin{gathered}
w_{t}=v_{x}, \\
v_{t}=\sigma(w)_{x}
\end{gathered}
$$


where $w=u_{x}$ is the strain and $v=u_{t}$ the velocity. The results of Part II have some relevance for this issue. In any case, the problem of dynamic stability for equilibria of (2.1) is at present intractable.

We consider instead the equation (1.1), a physically relevant regularization of (2.1), in which the stress includes a viscosity term proportional to the strain rate $u_{x t}$. Note that (1.1) and (2.1) have the same stationary solutions, with possibly discontinuous strain. In this part I we study the long-time behavior of solutions of an initial boundary value problem corresponding to a bar in a "soft" loading device. In such a device, one end of the bar is fixed, the other subjected to a prescribed load. Fixing $\mu=1$ for convenience, we consider the problem

$$
\begin{aligned}
u_{t t}= & \left(\sigma\left(u_{x}\right)+u_{x t}\right)_{x} \quad \text { for } 0<x<1, \quad t>0, \\
& u(0, t)=0, \\
& \left(\sigma\left(u_{x}\right)+u_{x t}\right)(1, t)=P \quad \text { for } t>0, \\
& u_{x}(x, 0)=u_{0}(x), \\
& u_{t}(x, 0)=u_{1}(x) \quad \text { for } 0 \leqq x \leqq 1 .
\end{aligned}
$$

These equations were first considered with monotone $\sigma$ by GREenBERG, MacCamy \& Mizel (1968) (see also Greenberg (1969) and Greenberg \& MaCCAMY (1970)) who showed that given smooth initial data, a smooth solution exists globally in time and decays to equilibrium at an exponential rate. DAFERMOS (1969) considered the more general equation $u_{t t}=\sigma\left(u_{x}, u_{x t}\right)$ with a parabolicity assumption and no monotonicity assumption in the first argument of $\sigma$, but with a rather restrictive growth condition. He showed that smooth solutions exist globally in appropriate Hölder classes (with $u(\cdot, t), u_{t}(\cdot, t)$ in $C^{2+\alpha}(0,1)$ ) and found that the velocity $u_{t}$ and the total stress $\sigma\left(u_{x}, u_{x t}\right)$ decay to zero as $t \rightarrow \infty$ in the Sobolev space $W^{1,2}(0,1)$. DAFERMOS also observed that the asymptotic behavior of the strain was an interesting issue, arguing that $u_{x}(x, t)$ in general need not approach a continuous function as $t \rightarrow \infty$.

ANDREws \& Ball (1982), following ANDrews (1980), established global existence of weak solutions to the problem (2.3) for initial data with $u_{x}(x, 0)$ in $L^{\infty}(0,1), u_{t}(x, 0)$ in $L^{2}(0,1)$, in particular admitting equilibria with discontinuous strain as data. Because of an a priori bound of ANDREws for the strain $u_{x}$, global existence could be guaranteed for data of limited energy for physically relevant $\sigma$ satisfying $\sigma(w) \rightarrow-\infty$ as $w \searrow 0$, under a mild sign condition. These authors showed that as $t \rightarrow \infty$, the velocity $u_{t} \rightarrow 0$ in $L^{2}$ and the elastic part of the stress $\sigma\left(u_{x}\right) \rightarrow P$ in $L^{2}$. They established weak-* convergence of the strain $u_{x}(x, t) \stackrel{*}{\rightarrow}$ $w_{\infty}(x)$ in $L^{\infty}$. But this left open the issue of whether in fact $\sigma\left(w_{\infty}(x)\right)=P$, that is, whether the strain converged to equilibrium.

\section{Plan of Part I}

The present treatment of the problem (2.3) will be mostly self-contained for the convenience of the reader, though it owes a sizable debt to the works above. In the first place, in $\S 3$ we present a simplified local existence theory for solutions 
of (2.3), based on the theory of abstract semilinear parabolic equations as presented by HENRY (1981) and the transformation in (2.9). As a consequence, we show that solutions of (2.3) enjoy a limited smoothing property: Given initial strain $u_{x}(x, 0)$ in $L^{\infty}$, and velocity $u_{t}(x, 0)$ in $L^{2}$, then for $t>0$ the total stress $\sigma\left(u_{x}\right)+\mu u_{x t}$ is $C^{1}$ in $x$ and $t$, and both sides of $\left(2.3_{1}\right)$ represent continuous functions. However, the strain $u_{x}$ and elastic stress $\sigma\left(u_{x}\right)$ need have no better regularity than $L^{\infty}$ in $x$. Indeed, initial discontinuities in $u_{x}$ must persist for all time without moving. (This fact was discovered by HoFF \& SMOLLER (1985) in the context of isothermal gas dynamics with viscosity.) However, solutions are smooth in $t$ if $\sigma$ is smooth. In the same abstract framework, we also establish existence of classical solutions with $C^{1}$ strain $u_{x}$, given $u_{x}(x, 0)$ in $C^{1}(0,1)$ and $u_{t}(x, 0)$ in $W^{1,2}(0,1)$ with $u_{t}(0,0)=0$.

Next, in $\S 4$ we begin to investigate the stability of equilibria having possibly discontinuous strain $u_{x}=w(x)$. On the basis of a spectral study we establish global existence and exponential decay to zero of small perturbations of equilibria satisfying the following dynamic stability criterion:

$$
\sigma^{\prime}(\tilde{w}(x)) \geqq \sigma_{0}>0, \quad \text { where } \sigma(\tilde{w}(x))=P .
$$

Perturbations in strain are required to be small in $L^{\infty}$, however, so that discontinuities in the asymptotic strain must also be present in the initial data. This requirement is relaxed in $\S 6$, at the expense of the exponential decay rate.

In $\S 5$ we investigate the asymptotic behavior of solutions for initial data $u_{x}(\cdot, 0)$ in $L^{\infty}$ and $u_{t}(\cdot, 0)$ in $L^{2}$. As in the results of ANDREWs \& BALL, if $\sigma(w)$ is defined for all $w$ and satisfies the mild condition (5.1), no further restrictions on the initial data are needed to ensure global existence of a solution with bounded strain. We show that solutions converge strongly to equilibrium, establishing the following asymptotic properties for the solution: As $t \rightarrow \infty$,

$$
\begin{gathered}
u_{t} \rightarrow 0 \quad \text { in } W^{1,2}(0,1) \\
\sigma\left(u_{x}\right)+u_{x t} \rightarrow P \quad \text { in } W^{2,2}(0,1) \\
u_{x}(x, t) \rightarrow w_{\infty}(x) \quad \text { boundedly a.e., where } \sigma\left(w_{\infty}(x)\right)=P \text { a.e. }
\end{gathered}
$$

Asymptotic states for smooth solutions are investigated in $\S 6$. Here equilibria $\tilde{u}(x)$ with possibly discontinuous strain $\tilde{w}(x)=\tilde{u}_{x}$ satisfying the stability criterion (2.4) are shown to be stable in a rather strong sense: If the perturbation in strain is small except on a set of small measure $\varepsilon$, then the solution will approach an equilibrium with strain equal to the unperturbed strain except perhaps on the same set of measure $\varepsilon$. It follows that a large class of smooth solutions have asymptotic limits with discontinuous strain.

It is important to relate the stability criterion obtained here to traditional energy criteria for stability of equilibria involving phase mixtures. (In the context of the present problem, such criteria have been discussed by ERICKSEN (1975) and JAMES (1980a).) Define a stored energy function

$$
W(w)=\int^{w} \sigma(s) d s .
$$


In the soft loading device of (2.3), the total energy

$$
\int_{0}^{1}\left[\frac{1}{2} u_{t}^{2}+W\left(u_{x}\right)-P u_{x}\right](y, t) d y
$$

is a decreasing function of time. One expects the total stored energy functional

$$
I(u)=\int_{0}^{1}\left[W\left(u_{x}\right)-P u_{x}\right](y, t) d y
$$

to approach a minimum. In the present model, a typical function $W(w)-P w$ as obtained from Fig. 1 might be as in Fig. 2, having two local minima $\alpha_{-}$and $\beta_{-}$.

The problem of minimizing $I(u)$ in (2.7) is a standard one in the calculus of variations. Much attention has traditionally been focussed on absolute minima, and in particular on strong minimizers $\tilde{u}(x)$. A strong minimizer satisfies $I(\tilde{u}+v) \geqq I(\tilde{u})$ for all absolutely continuous $v(x)$ with $\|v\|_{L^{\infty}}$ small. At a strain discontinuity the Weierstrass-Erdmann corner conditions for a strong minimizer imply that $W\left(\tilde{u}_{x}\right)-P \tilde{u}_{x}$ is continuous across the discontinuity. Thus in the situation of Fig. 2, an equilibrium containing a phase discontinuity from $\alpha_{-}$to $\beta_{-}$ cannot be a strong minimizer unless the stored energy density is the same in the two phases, that is, $W\left(\alpha_{-}\right)-P \alpha_{-}=W\left(\beta_{-}\right)-P \beta_{-}$. This implies that the stress level $P$ in Figure 1 is at the Maxwell line, meaning that the two bounded regions in Figure 1 delineated by the curves $\sigma=P$ and $\sigma=\sigma(w)$ have equal area.

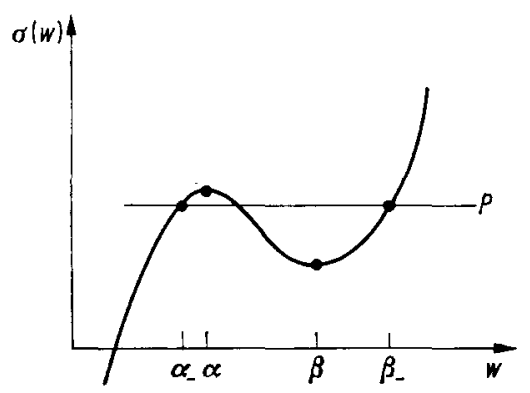

Fig. 1. Stress-strain relation

Regardless of these facts, it is clear that an equilibrium $\tilde{u}(x)$ for $(2.3)$, whose strain $\tilde{u}_{x}$ takes both values $\alpha_{-}$and $\beta_{-}$, can easily satisfy the condition (2.4) for dynamic stability even if the stored-energy densities at $\alpha_{-}$and $\beta_{-}$differ. Indeed, the condition (2.4) is the condition in the calculus of variations that the second variation $\delta^{2} I(u)$ of the functional $I(u)$ be strictly positive, and it implies that $\tilde{u}(x)$ is a weak relative minimizer of $I(u)$, satisfying $I(\tilde{u}+v) \geqq I(\tilde{u})$ whenever both $\|v\|_{L^{\infty}}$ and $\left\|v_{x}\right\|_{L^{\infty}}$ are small.

To paraphrase the conclusions, then: A positive second variation implies dynamic stability in the viscoelastic problem (2.3). The property of being a strong minimizer, on the other hand, is not necessary for dynamic stability in (2.3). It is common to call a state of strain metastable (respectively stable) if it provides 


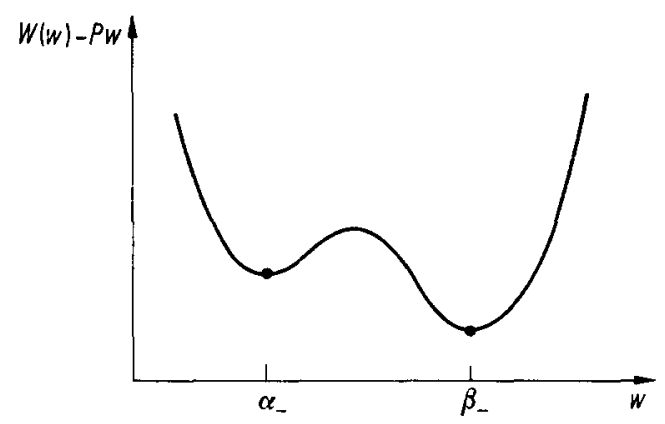

Fig. 2. Stored-energy density

a local (respectively global) minimum of the stored energy density $W(w)-P w$. Thus for (2.3) metastable states can coexist with stable states in stable asymptotic limits of smooth solutions.

Part I concludes with an application of the stability analysis of $\S 6$ to a hypothetical load-deformation experiment. In $\S 7$, we consider a bar-placed in a soft loading device, in which the end load $P$ is raised or lowered in discrete increments, and "fluctuations" are imposed on the displacement and velocity at discrete intervals of time. The fluctuations are regarded as a model accounting for unknown physical influences on the bar. We illustrate how hysteresis occurs dynamically under suitable restrictions on the load increments and fluctuations. The process must proceed slowly, but we do not assume it is "quasistatic"; inertial effects are fully taken into account. If the load is held constant near a level of transition between phases and if certain assumptions are made about the fluctuations, also a "creep" phenomenon can occur, as inhomogeneities induced by localized fluctuations of small energy cause a slow change in the overall length of the bar.

We conclude this section by introducing the main tools for studying (2.3). A certain transformation of the equations will be very useful throughout: Suppose we have a (smooth) solution of (2.3), and introduce quantities

$$
\begin{gathered}
p(x, t)=\int_{1}^{x} u_{t}(y, t) d y \\
q(x, t)=u_{x}(x, t)-p(x, t) .
\end{gathered}
$$

The solution $u$ may be recovered from

$$
u(x, t)=\int_{0}^{x}(p+q)(y, t) d y .
$$

Then $p$ and $q$ form a solution to the problem

$$
\begin{aligned}
& p_{t}=p_{x x}+\sigma(p+q)-P \quad \text { for } 0<x<1, \quad t>0 \\
& q_{t}=-\sigma(p+q)+P \\
& \quad p_{x}(0, t)=0, \quad p(1, t)=0 \quad \text { for } t>0 \\
& p(x, 0)=p_{0}(x), \quad q(x, 0)=q_{0}(x) \quad \text { for } 0 \leqq x \leqq 1 .
\end{aligned}
$$


I note that the "modified strain" $q$ is the same (up to sign) as that used by ANDREws (1980) to establish global existence of solutions to (2.3) based on the ordinary differential equation that $q$ satisfies. In equilibrium $q$ is equal to the strain $u_{x}$. The "velocity potential" $p$ also has an interesting property. Write the viscoelastic equation in (2.3) as a system in the standard way: with the total stress

$$
S=\sigma\left(u_{x}\right)+u_{x t}-P
$$

we have

$$
\begin{aligned}
& w_{t}=v_{x} \\
& v_{t}=S_{x}
\end{aligned}
$$

where

Then

$$
w=u_{x}, \quad v=u_{t}
$$

$$
v=p_{x}, \quad S=p_{t} \text {. }
$$

The fact that $S$ is the time derivative of $p$ will be important for establishing its regularity. I remark that the structure of (2.9), a parabolic partial differential equation coupled to an ordinary differential equation, illustrates why the viscoelastic equation may be viewed as an abstract parabolic equation (even though the viscosity in (2.11) is "degenerate"), and exhibits explicitly a zero-speed characteristic in equation (1.1), associated with the ordinary differential equation for $q$ in (2.9).

The transformation above is our main tool for the existence and regularity theory. Two other ingredients are important in the study of asymptotic behavior and stability: the energy identity and a lemma on invariant intervals for the ordinary differential equation in (2.9). With $W(w)$ the stored-energy function from (2.6), the energy identity for (2.3) reads

$$
\begin{aligned}
\int_{0}^{1}\left(\frac{1}{2} u_{t}^{2}\right. & \left.+W\left(u_{x}\right)-P u_{x}\right)(y, t) d y+\int_{0}^{t} \int_{0}^{1} u_{x t}^{2}(y, \tau) d y d \tau \\
& =\int_{0}^{1}\left(\frac{1}{2} u_{t}^{2}+W\left(u_{x}\right)-P u_{x}\right)(y, 0) d y
\end{aligned}
$$

in terms of $p$ and $q$,

$$
\begin{gathered}
\int_{0}^{1} \frac{1}{2} p_{x}^{2}(x, t) d y+\int_{0}^{1}(W(p+q)-P(p+q))(y, t) d y+\int_{0}^{t} \int_{0}^{1} p_{x x}^{2}(y, \tau) d y d \tau \\
=\int_{0}^{1} \frac{1}{2} p_{x}^{2}(y, 0) d y+\int_{0}^{1}(W(p+q)-P(p+q))(y, 0) d y .
\end{gathered}
$$

Provided $W(w)-P w$ is bounded below, the energy identity yields an a priori bound on the kinetic energy, hence on $p$ pointwise, by the estimate

$$
|p(x, t)|^{2} \leqq(1-x) \int_{x}^{1} p_{x}^{2}(y, t) d y \leqq \int_{0}^{1} u_{t}^{2}(y, t) d y,
$$


valid since $p(1, t)=0$. Control on $q$ is to be achieved pointwise in $x$ via the following easily proved lemma:

Lemma 2.1 (Invariant intervals). Consider the ordinary differential equation

$$
q^{\prime}(t)=-\sigma(p(t)+q(t))
$$

where $\sigma(w)$ is a locally Lipschitz-continuous function on an interval in $\mathbb{R}$ and $p(t)$ is locally Lipschitz-continuous in $t$. Assume that $\varepsilon>0$ and $w_{-}<w_{+}$exist such that

i) $|p(t)|<\varepsilon \quad$ for $0 \leqq t \leqq T \leqq \infty$

ii) $\sigma\left(w_{-}+w\right)<0<\sigma\left(w_{+}+w\right) \quad$ whenever $|w|<\varepsilon$.

Then the interval $\left[w_{-}, w_{+}\right]$is positively invariant for the ordinary differential equation on the time interval $[0, T]$. That is if $q\left(t_{0}\right) \in\left[w_{-}, w_{+}\right]$and $0 \leqq t_{0} \leqq T$, then $q(t) \in\left[w_{-}, w_{+}\right]$whenever $t_{0} \leqq t \leqq T$ (Figure 3 ).

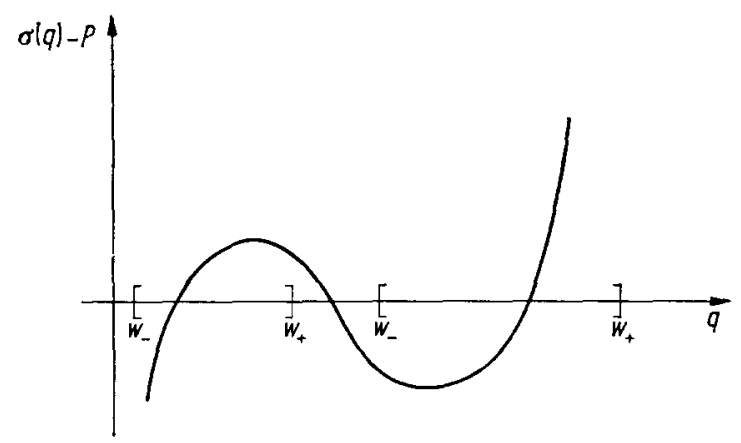

Fig. 3. Invariant intervals for modified strain

This lemma yields the following "principle of invariant phases": The strain in (2.3) cannot change phase at any point, provided only that the total kinetic energy of the bar remain small. The stability established in $\$ 6$ depends on controlling the conversion of potential energy to kinetic energy in a situation where there may be smooth transition layers joining "metastable" and "stable" phases.

\section{Local Existence and Regularity}

We consider the problem (2.3) (or equivalently, (2.9); see 3.2, 3.3 below). We assume that the stress $\sigma(w)$ is defined on an open interval $J \subseteq \mathbb{R}$, and will consider cases where a) $\sigma(w)$ is locally Lipschitz-continuous, and b) $\sigma(w)$ is $C^{\mathbf{1}}$ with $\sigma^{\prime}(w)$ locally Lipschitz-continuous. In the latter case we obtain greater regularity and existence of classical solutions for (2.3). In $\S 5$ below, we will impose an additional condition (e.g., see (5.1)) on $\sigma(w)$ to establish global existence for initial data of unrestricted energy. 
The basic existence theorem concerns the transformed problem (2.9):

Theorem 3.1. (1) (Local existence) Suppose $\sigma(w)$ is locally Lipschitz-continuous. Assume that $q_{0} \in L^{\infty}(0,1)$ and $p_{0} \in W^{1,2}(0,1)$ with $p_{0}(1)=0$, and suppose $p_{0}(x)+q_{0}(x) \in J_{0}$ a.e. where $\bar{J}_{0} \subset J$. Then there exists $T>0$ so that a unique (strong) solution of (2.9) exists for $0 \leqq t \leqq T$, with

$$
p \in C\left([0, T], W^{1,2}\right) \cap C^{1}\left((0, T], L^{2}\right) \cap C\left((0, T], W^{2,2}\right)
$$

and $p_{x}(0, t)=0=p(1, t)$ for $t>0$, and

$$
q \in C^{1}\left([0, T], L^{\infty}\right) \text {. }
$$

(2) (Regularity) For any $\delta>0$ and $v<1 / 2$, the solution above has for some $\beta>0$,

$$
p_{t}=S \in C^{\beta}\left([\delta, T], C^{1, v}\right)
$$

with $S_{x}(x, t)=0=S(1, t)$ for $t>0$,

$$
q_{t}, p_{x x} \in C^{\beta}\left([\delta, T], L^{\infty}\right) .
$$

(Here $C^{\beta}$ is a space of Hölder-continuous functions, and $C^{1, v}$ is the space of $C^{1}$ functions on $[0,1]$ with Hölder-continuous derivative, with exponent $v$.) If $\sigma(w)$ is $C^{1}$ with $\sigma^{\prime}(w)$ locally Lipschitz-continuous, then also

$$
\begin{gathered}
p_{t t}=S_{t} \in C^{\beta}\left([\delta, T], C^{1, \nu}\right), \\
p_{t x x}=S_{x x} \in C^{\beta}\left([\delta, T], L^{\infty}\right) .
\end{gathered}
$$

Furthermore, if $\sigma(w)$ is $C^{r}, r \geqq 2$ (respectively, analytic), then $p$ and $q$ are $C^{r}$ in $t$ (respectively, analytic in $t$ ) with values in $W^{1,2}$ and $L^{\infty}$, respectively, for $t>0$.

The result above yields "almost classical" solutions to the problem (2.3), obtained from $u(x, t)=\int_{0}^{x}(p+q)(y, t) d y$ :

Corollary 3.2. Suppose $\sigma(w)$ is locally Lipschitz-continuous. Assume $u_{0} \in L^{\infty}(0,1)$ with $u_{0}(x) \in J_{0}$ a.e., $\bar{J}_{0} \subset J$, and $u_{1} \in L^{2}(0,1)$. Then a unique solution $u(x, t)$ to (2.3) exists for $t$ in some interval $[0, T], T>0$, with (for any $\delta>0, v<\frac{1}{2}$, and some $\beta>0$ )

$$
\begin{gathered}
u \in C\left([0, T], W^{1, \infty}\right), \quad u(0, t)=0, \\
u_{x t} \in C^{\beta}\left([\delta, T], L^{\infty}\right), \\
u_{t t} \in C^{\beta}\left([\delta, T], C^{y}\right), \\
S=u_{x t}+\sigma\left(u_{x}\right)-P \quad \text { for a.e. } x .
\end{gathered}
$$

If $\sigma(w)$ is $C^{1}$ with $\sigma^{\prime}(w)$ locslly Lipschitz-continuous, then also

$$
u_{t t} \in C^{\beta}\left([\delta, T], W^{1, \infty}\right) .
$$

Thus both $u_{t t}$ and $S_{x}$ are at least Hölder continuous for $t>0$, with $u_{t t}=S_{x}$, $u(0, t)=0=S(1, t)$. 
Remark 3.3. The weak solutions of (2.3) constructed by ANDREws \& BALL with initial data as in 3.2 yield, by the transformation (2.8), weak solutions of (2.9) which are solutions pointwise a.e., with $p_{x}, q \in C\left([\delta, T], L^{\infty}\right)$ for any $\delta>0$. By uniqueness, the solutions agree with those of 3.1. So the weak solutions of ANDREWS \& BALL in fact have the regularity indicated in 3.2 above. (To establish the correspondence, write the weak form of the equation (2.3) in terms of test functions $\psi$ of the form $\psi=\phi_{x}$. It follows that (2.9) holds in the sense of distributions, for $L^{\infty}$ functions.)

At the end of this section we will discuss the local existence of classical solutions of (2.3) (having $u_{x}$ in $C^{1}[0,1]$ ) by modifying a bit the framework we employ below. We will also make a remark concerning the persistence of initial discontinuities in $u_{x}$ in (2.3). Now, I present a fact that will make the study of solutions of the ordinary differential equation $\left(2.9_{2}\right)$ technically a bit easier:

Corollary 3.4. Let $(p, q)$ be a solution of (2.9) as given by Theorem 3.1. Then there is a fixed subset of $[0,1]$ (of full measure) depending on the solution, such that, for those $x$ in the subset, $q(x, t)$ satisfies $\left(2.9_{2}\right)$ for all $t, 0<t \leqq T$. Thus $q$ is a classical solution of the ordinary differential equation $\left(2.9_{2}\right)$ for $t>0$, for a.e. $x$ in $(0,1)$.

Proof of 3.1. My approach is to apply standard results as presented by HENRY (1981) concerning the abstract initial value problem in a Banach space $X$,

$$
\begin{gathered}
z_{t}+A z=f(z) \quad t>0 \\
z(0)=z_{0}
\end{gathered}
$$

and deduce pointwise properties of the solution from embedding theorems. In our application, $z=(p, q)$ and the space $X=\left(L^{2}, L^{\infty}\right)$. The operator $A=\left(\begin{array}{rr}-\Delta & 0 \\ 0 & 0\end{array}\right)$ where $\Delta=\partial^{2} / \partial x^{2}$. For the boundary conditions in (2.9) the domain of $A$ is taken as $D(A)=\left(D(A), L^{\infty}\right)$ where

$$
D(\Delta)=\left\{p \in W^{2,2}(0,1) \mid p_{x}(0)=0=p(1)\right\} .
$$

We also write $D(\Delta)=W_{b}^{2,2}$. Now $-\Delta$ is a self-adjoint, densely defined, positive operator on $L^{2}(0,1)$, so $A$ is sectorial on $X$. For the sequel, we need to identify the domain of the square root of $A, X^{\frac{1}{2}}=D\left(A^{\frac{1}{2}}\right)$ :

Lemma.

$$
X^{\frac{1}{2}}=\left(W_{b}^{1,2}, L^{\infty}\right)
$$

where

$$
W_{b}^{1,2}=\left\{\left(p \in W^{1,2}(0,1) \mid p(1)=0\right\}\right.
$$

Proof. It is clear that $X^{\frac{1}{2}}=\left(D\left((-\Delta)^{\frac{1}{2}}\right), L^{\infty}\right)$. Denote $D\left((-\Delta)^{\frac{1}{2}}\right)$ by $Y$. The space $Y$ is the closure of $D(\Delta)$ in the norm given for $u \in D(A)$ by

$$
\|u\|_{\frac{1}{2}}^{2}=\left\|(-\Delta)^{\frac{1}{2}} u\right\|_{L^{2}}^{2}=(-\Delta u, u)=\int_{0}^{1}-u_{x x} u=\int_{0}^{1} u_{x}^{2} .
$$


Therefore $Y$ is the closure of $D(\Delta)$ in the $W^{1,2}$ norm, so $Y \subset W^{1,2}$ and $u(1)=0$ for $u \in Y$. Thus $Y \subset W_{b}^{1,2}$. Conversely, if $u \in W_{b}^{1,2}$ we may approximate $u$ by $u_{n} \in D(\Delta)$ as follows: $u(x)=\int_{1}^{x} u_{x}$, so let $v=u_{x}$ be approximated by smooth $v_{n}$ in $L^{2}$ with $v_{n}(0)=0$. With $u_{n}=\int_{1}^{x} v_{n}$ we have $u_{n} \in D(\Lambda), u_{n} \rightarrow u$ in $W^{1,2}$.

The last thing to check before applying the abstract theory is the smoothness of the map

$$
(p, q) \rightarrow(\sigma(p+q)-P,-\sigma(p+q)+P)
$$

from $\left(W_{b}^{1,2}, L^{\infty}\right)$ to $\left(L^{2}, L^{\infty}\right)$. Since the inclusions

$$
W_{b}^{1,2} \hookrightarrow L^{\infty} \hookrightarrow L^{2}
$$

are analytic (linear), the smoothness of the map above is the same as the smoothness of the map $w(x) \rightarrow \sigma(w(x))$ on $L^{\infty}$. But it is clear that if $\sigma$ is Lipschitzcontinuous, $C^{r}$ or analytic on a closed interval $[a, b]$, this map is Lipschitz-continuous, $C^{r}$ or analytic on $\left\{w \in L^{\infty}(0,1) \mid w(x) \in[a, b]\right.$ a.e. $]$.

We now invoke the theorem of HENRY 3.3.3 (1981) to deduce existence of a unique local solution of (3.1), given $z_{0}=\left(p_{0}, q_{0}\right) \in X^{\frac{1}{2}}$ such that $\left(p_{0}+q_{0}\right)$ $(x) \in J$ a.e. This solution has the following regularity at this point (see HeNRY 3.2.1 and the proof of 3.3.3):

$$
z(t) \in C\left([0, T], X^{\frac{1}{2}}\right) \cap C^{1}((0, T], X) \cap C((0, T], D(A)) .
$$

(Continuity into $D(A)$ follows because $z$ satisfies $\left(3.1_{1}\right)$ for $t>0$.) Taking components of $z$ establishes part (1) of Theorem 3.1. That $q_{t} \in C\left([0, T], L^{\infty}\right)$ follows from the equation $(2.9)_{2}$. Note at this point that $p_{t}$ exists as a distribution with $p_{t}(t)$ in $L^{2}$, and $\left(2.9_{1}\right)$ holds for each $t>0$ in the sense of $L^{2}$ functions. Later in $\S 5$ we will need more precise bounds for $z(t)$ in the spaces above.

The next step is to deduce greater regularity of the solution for $t>0$ from the conclusions of HENRY 3.5.2 which imply that

$z_{t}$ is locally Hölder-continuous from $(0, T]$ to $X^{\gamma}$, for any $\gamma<1$.

Now clearly $X^{\gamma}=\left(D\left((-\Delta)^{\gamma}\right), L^{\infty}\right)$, and it is true that

$$
D\left((-\Delta)^{\gamma}\right) C C^{1 v} \quad \text { if } 2 \gamma>v+3 / 2 .
$$

(This follows from the conclusions of HeNRY 1.6.1). Since $p_{x x}=p_{t}+q_{t}$, the first part of (2) follows by taking components.

Suppose now that $\sigma(w)$ is $C^{1}$ with $\sigma^{\prime}(w)$ locally Lipschitz-continuous. We claim that then $S=p_{t}$ is a strong solution of

$$
\begin{gathered}
S_{t}=S_{x x}+\sigma(p+q)_{t} \quad \text { for } t>0 \\
S_{x}(0, t)=0=S(1, t) .
\end{gathered}
$$

This is true because from the theorems of HENRY 3.4.4 and 3.4.6 we know $S$ is a mild solution of this equation, and we can verify that the map $t \rightarrow \sigma(p+q)$, is locally Hölder-continuous for $t>0$ into $L^{\infty} \subset L^{2}$, and so from the conclusions 
of HENRY 3.2.2, $S$ is a strong solution on $[\delta, T]$ for any $\delta>0$. We conclude that

$$
S=p_{t} \in C^{1}\left((\delta, T], L^{2}\right) \cap C\left((\delta, T], W_{b}^{2,2}\right) .
$$

But now the map $(t, S) \rightarrow \sigma^{\prime}(p+q)\left(S+q_{t}\right)$ is clearly locally Lipschitz-continuous from $\mathbb{R} \times W^{1,2}$ to $L^{\infty}$, so by applying again the statements of HENRY 3.5.2 we may conclude that $S_{t}$ is locally Hölder-continuous into $D\left((-\Delta)^{\gamma}\right)$ for any $\gamma<1$. Then from equation (3.2) it follows that $S_{x x}$ is locally Hölder-continuous into $L^{\infty}$.

The remarks concerning the case when $\sigma(w)$ is $C^{r}$ or analytic follow from the theorems of HENRY 3.4.4 and 3.4.6. This finishes the proof of Theorem 3.1.

Proof of Corollary 3.2. Given the solution $z=(p, q)$ from Theorem 3.1, let $u(x, t)=\int_{0}^{x}(p+q)(y, t) d y$. Then $u_{x t}=p_{t}+q_{t}=p_{x x}$ is locally Hölder-continuous into $L^{\infty}$ and is $C^{1}$ into $L^{2}$ for $t>0$, so $u_{t}=\int_{0}^{x} p_{x x}=p_{x}$, whence $u_{t t}=$ $p_{x t}=S_{x}$ as distributions. The regularity of $u_{t t}$ then follows from the regularity of $S$.

Proof of Corollary 3.4. Because $q(t)$ satisfies $\left(2.9_{2}\right)$ in $L^{\infty}, q \in C^{1}\left([0, T], L^{\infty}\right)$. Then, in $L^{\infty}$

$$
q(t)=q_{0}+\int_{0}^{t} q_{t}(s) d s=q_{0}-\int_{0}^{t}(\sigma(p+q)(s)-P) d s
$$

so long as the solution exists. The integral converges as a Riemann integral in $C\left([0, T], L^{\infty}\right)$. Fixing a sequence of partitions of $(0, \infty)$ with norm approaching zero, we may delete a countable union of sets of measure zero in $(0,1)$ to guarantee that on a set of full measure in $x$, the integral converges in the sup norm. For $x$ in this set, then,

$$
q(x, t)=q(x, 0)+\int_{0}^{t} \sigma(p+q)(x, s) d s
$$

so $q(x, t)$ is Lipschitz-continuous in $t$.

We have proved that the solution of the problem (2.3) enjoys some limited spatial smoothing: The velocity $u_{t}$ improves from $L^{2}$ at $t=0$ to $W^{1, \infty}$ for $t>0$, and the total stress $S$ gains from $W^{-1,2}$ (roughly) at $t=0$ to $W^{2, \infty}$ for $t>0$, if $\sigma(w)$ is smooth. But whereas the solution is smooth in time if $\sigma(w)$ is smooth, we observe that the strain $u_{x}$ is not smoothed in space:

Proposition 3.5. (Persistence of strain discontinuities). Suppose $x_{0} \in(0,1)$ is a point of discontinuity of the initial strain $u_{0}(x)$ in (2.4). Then $x_{0}$ is a point of discontinuity of $u_{x}(x, t)$ for the solution $u(x, t)$ from Corollary 3.2, for any $t \geqq 0$ fixed, so long as the solution exists.

(An observation of this type has been made by Hoff \& SMOLLER (1985) for the system of isothermal gas dynamics.) 
For the proof, recall that $u_{x}==p+q$. Now $p(x, t)$ is absolutely continuous in $(x, t)$ for $t \geqq 0$, so $x_{0}$ is a point of discontinuity of $q_{0}(t)$. The ordinary differential equation $\left(2.9_{2}\right)$ now implies $x_{0}$ remains a point of discontinuity of $q(x, t)$ for any fixed $t>0$. For if not, if $q\left(x, t_{0}\right)$ were continuous at $x_{0}$ for some $t_{0}>0$, then, running the ordinary differential equation backwards from $t=t_{0}$ to $t=0$, we see that continuous dependence on parameters in ordinary differential equations makes $q(x, 0)$ continuous at $x_{0}$, a contradiction.

Solutions of (2.3) also preserve their initial smoothness, as established by Dafermos (1969) and Greenberg MacCamy \& Mizel (1968). Thus sufficiently smooth data yield classical solutions, for which every term in (2.3) is continuous. By a simple variant of the argument for Theorem 3.1, we can obtain such classical solutions:

Theorem 3.6 (Classical solutions). Suppose $\sigma(w)$ is $C^{1}$, with $\sigma^{\prime}(w)$ locally Lipschitzcontinuous. Assume $u_{0} \in C^{1}[0,1] \quad u_{0}(x) \in J_{0}$ a.e., $\vec{J}_{0} \subset J$, and $u_{1} \in W^{1,2}[0,1]$ with $u_{1}(0)=0$. Then for some $T>0$, a classical solution $u(x, t)$ exists for $(2.3)$ for $0 \leqq t \leqq T$, having

$$
u \in C\left([0, T], C^{2}\right),
$$

$u_{t t}, u_{x f x}$ continuous in $(x, t)$ for $t>0$.

Proof of 3.6. Again we consider (2.9) with initial data $p_{0}(x)=\int_{1}^{x} u_{1}(y) d y$, $q_{0}(x)=u_{0}(x)-p_{0}(x)$, so that $p_{0} \in W_{b}^{2,2}, q_{0} \in C^{1}$. As in the proof of 3.1, we seek to apply the theorem of HENRY 3.3 .3 to obtain local existence. Redefine $X=\left(L^{2}, C^{1}\right)$. The operator $A$ generates an analytic semigroup on $X$. We seek to obtain solutions in $C\left([0, T], X^{\alpha}\right)$ for $3 / 4<\alpha<1$. Since $X^{\alpha}=\left(D\left((-\Delta)^{\alpha}\right), C^{1}\right)$ and $W_{b}^{2,2}=D\left((-A)^{1}\right) C D\left((-A)^{\alpha}\right)$, we have $\left(p_{0}, q_{0}\right) \in X^{\alpha}$, so all we must show is that

$$
f(p, q)=(\sigma(p+q)-P,-\sigma(p+q)+P)
$$

is locally Lipschitz-continuous from $X^{\alpha}$ to $X$. But for $\alpha>\frac{3}{4}$,

$$
D\left((-\Delta)^{\alpha}\right) C C^{1} C L^{2} \text {. }
$$

Thus the desired fact follows because the map $w(x) \rightarrow \sigma(w(x))$ is locally Lipschitz-continuous from $C^{1}$ to $C^{1}$. Now from the theorems of HENRY 3.5.2 the local solution

$$
(p, q) \in C\left([0, T], X^{\alpha}\right) \cap C^{1}\left((0, T], X^{\gamma}\right)
$$

for any $\gamma<1$. In particular, the functions $p_{t x}, q_{t x}$ are continuous in $(x, t)$ for $t>0$ and so with $u(x, t)=\int_{0}^{x}(p+q)(y, t) d y$, we find that the equation

$$
u_{t t}=\sigma^{\prime}\left(u_{x}\right) u_{x x}+u_{x t x}
$$

is satisfied, with each term continuous in $(x, t)$ for $t>0$. 
The proof above suggests that in Theorem 3.1, where $X=\left(L^{2}, L^{\infty}\right)$, we can find local solutions of (2.9) whenever $\left(p_{0}, q_{0}\right) \in X^{\alpha}$ for $1 / 4<\alpha<1$. For $\alpha<\frac{1}{2}$, this would admit initial data with infinite kinetic energy $\frac{1}{2} \int p_{x}^{2}$. (The kinetic energy would be finite at any positive time, however, since one would have $p \in C\left((0, T], W^{2,2}\right)$.)

\section{Exponentially Stable States}

In this section, by means of linearized stability analysis, we identify equilibria of the problem (2.9) which are stable with exponential decay rate for perturbations small in the space $X^{\frac{1}{2}}=\left(W_{b}^{1,2}, L^{\infty}\right)$. The criterion for stability, (2.4), is positivity of the second variation of the stored-energy functional (2.7). Equilibria with negative second variation are unstable (see 4.2 below).

Theorem 4.1. (Exponential stability). Suppose $\sigma(w)$ is $C^{1}$ and $\sigma^{\prime}(w)$ is locally Lipschitz-continuous. Let $(\tilde{p}, \tilde{q})=(0, \tilde{w}(x))$ be an equilibrium solution of $(2.9)$, and assume

$$
\sigma(\tilde{w}(x))=P, \quad \sigma^{\prime}(\tilde{w}(x)) \geqq \sigma_{0}>0 \text { a.e. }
$$

Then for any $\delta<\min \left\{\pi^{2} / 8, \sigma_{0}\right\}$, a unique solution $(p, q)$ of (2.9) exists globally for $t>0$ and satisfies. for some $C_{0}>0$,

$$
\|p(t)\|_{W^{1,2}} \leqq C_{0} e^{-\delta t}, \quad\|q(t)-\tilde{w}\|_{L^{\infty}} \leqq C_{0} e^{-\delta t},
$$

provided that $\left\|p_{0}\right\|_{W^{1,2}}$ and $\left\|q_{0}-\tilde{w}\right\|_{L^{\infty}}$ are sufficiently small, with $p_{0}(1)=0$. Here $\cdot \pi^{2} / 8=\beta_{0} / 2$ where $\beta_{0}$ is the first eigenvalue of -4 on $(0,1)$ with boundary conditions $\left(2.9_{3}\right)$.

Proof. We shall apply the statements of HenRY 5.1.1 in the abstract framework of the proof of 3.1. Let $\tilde{z}=(0, \tilde{w}), z=(p, q)$. For the abstract equation (3.1) we have

$$
f(z+\tilde{z})=f(\tilde{z})+B \dot{z}+g(z)
$$

where $f(\tilde{z})=0$,

and

$$
B z=B(p, q)=\sigma^{\prime}(\tilde{w}(x))\left(\begin{array}{rr}
1 & 1 \\
-1 & -1
\end{array}\right)\left(\begin{array}{l}
p \\
q
\end{array}\right)
$$

$$
g(z)=\left(\sigma(p+q+\tilde{w})-\sigma(\tilde{w})-\sigma^{\prime}(\tilde{w})(p+q)\right)\left(\begin{array}{r}
1 \\
-1
\end{array}\right) .
$$

The smoothness assumption and (2.4) imply that the essential range of $\tilde{w}(x)$ is a finite set of values. Applying Taylor's theorem, one concludes that if $(p, q)$ is small in $X^{\frac{1}{2}}=\left(W_{b}^{1,2}, L^{\infty}\right)$, then for some $C$,

$$
\|g(z)\|_{x} \leqq C\|p+q\|_{L^{\infty}}^{2} .
$$

The linear map $B$ is bounded on $\left(L^{\infty}, L^{\infty}\right)$, so is bounded from $X^{\frac{1}{2}}$ to $X$. 
Our main work is to check the last hypothesis of HenRy 5.1.1: to show that the spectrum of the operator $A-B$ on $X$ lies in a half plane

$$
\left\{\lambda \in \mathbb{C} \mid \operatorname{Re} \lambda \geqq \min \left(\sigma_{0}, \beta_{0} / 2\right)\right\} .
$$

This shall be accomplished in two stages. First, we will show that the essential spectrum of $A-B$ (the spectrum with discrete eigenvalues of finite multiplicity deleted) is concentrated on the essential range of $\sigma^{\prime}(\tilde{w}(x))$. Second, we show that no eigenvalue $\lambda$ can satisfy $\operatorname{Re} \lambda<\min \left(\sigma_{0}, \beta_{0} / 2\right)$. The first step will be achieved using the invariance of the essential spectrum under relatively compact perturbation (see Henry, ch. 5, A. 1). The second step employs an energy method.

We proceed with step one. Decompose $B$ as $B_{p}+B_{q}$ where

$$
B_{p}=\sigma^{\prime}(\tilde{w})\left(\begin{array}{rr}
1 & 0 \\
-1 & 0
\end{array}\right), \quad B_{q}=\sigma^{\prime}(\tilde{w})\left(\begin{array}{rr}
0 & 1 \\
0 & -1
\end{array}\right)
$$

Then whenever $\lambda \in \mathbb{C}$ is not contained in the spectrum $\operatorname{sp}(-\Delta)$ or the essential range of $\sigma^{\prime}(\tilde{w}(x))$, an explicit representation for the resolvent of the operator

is

$$
A-B_{q}=\left(\begin{array}{rr}
-\Delta & -\sigma^{\prime} \\
0 & \sigma^{\prime}
\end{array}\right)
$$

$$
\left(\lambda-A+B_{q}\right)^{-1}=\left[\begin{array}{cc}
(\lambda+\Delta)^{-1} & -(\lambda+\Delta)^{-1} \sigma^{\prime}\left(\lambda-\sigma^{\prime}\right)^{-1} \\
0 & \left(\lambda-\sigma^{\prime}\right)^{-1}
\end{array}\right] .
$$

On the interval $(0,1)$ with boundary conditions $\left(2.9_{3}\right), \operatorname{sp}(-\Delta)$ consists of discrete positive eigenvalues, so the essential spectrum of $A-B_{q}$ is the essential range of $\sigma^{\prime}(\tilde{w}(x))$.

Now we claim that $B_{p}\left(-A+B_{q}\right)^{-1}$ is compact on $X$. From the representations above, it suffices to verify to that $(-A)^{-1}$ is bounded from $L^{2}$ to $W^{1, \infty}(0,1)$, so that it is compact from $L^{2}$ to $L^{\infty}$. For this, we just verify that the Green's function for $-\mathcal{A}$, which satisfies

satisfies also

$$
\left(-\Delta^{-1} f\right)(x)=\int_{0}^{1} G(x, y) f(y) d y
$$

$$
\int_{0}^{1} G_{x}^{2}(x, y) d y<C \text { independent of } x .
$$

But explicitly, $G(x, y)=1-y$ if $x<y, 1-x$ if $x>y$, so this holds.

Now applying the statements of HENRY ch. 5, A. 1, we conclude that any point $\lambda$ in the spectrum of $A-B$ is either in the essential range of $\sigma^{\prime}(\tilde{w}(x)$ ) (a finite set), or is an eigenvalue on $X$.

We proceed with step two. Suppose $\lambda \in \mathbb{C}$ is an eigenvalue of $A-B$ not in the essential range of $\sigma^{\prime}(\tilde{w}(x))$, with eigenfunction $z(x)=(p, q)(x)$. Then the real and imaginary parts of $p$ are in $W_{b}^{2,2}=D(-\Delta)$, and we have

$$
\begin{gathered}
\lambda p+p_{x x}+\sigma^{\prime}(\tilde{w})(p+q)=0 \\
\lambda q-\sigma^{\prime}(\tilde{w})(p+q)=0 .
\end{gathered}
$$


Solve for $q$ in terms of $p$ and substitute, obtaining

where

$$
p_{x x}+\beta\left(\lambda, \sigma^{\prime}(\tilde{w})\right) p=0
$$

$$
\beta(\lambda, s)=\frac{\lambda^{2}}{\lambda-s}
$$

Multiply by $\bar{p}$ and integrate by parts, obtaining

$$
\int_{0}^{1}\left[\beta\left(\lambda, \sigma^{\prime}(w(x))\right)|p(x)|^{2}-\left|p_{x}(x)\right|^{2}\right] d x=0 .
$$

Now apply the sharp Poincaré inequality valid for any $p \in W^{1,2}, p(1)=0$ :

$$
\int_{0}^{1}\left|p_{x}\right|^{2} d x / \int_{0}^{1}|p|^{2} d x \geqq \beta_{0}=\pi^{2} / 4
$$

Then we have

$$
0 \geqq \int_{0}^{1}\left[\beta_{0}-\beta\left(\lambda, \sigma^{\prime}(\tilde{w}(x))\right)\right]|p(x)|^{2} d x .
$$

Note that in this inequality, $\beta$ may take complex values. It is clear that if $\lambda$ is real with $\lambda<\sigma_{0}$, then $\beta\left(\lambda, \sigma^{\prime}(\tilde{w})\right)<0$ a.e., which forces $p=0$, so $\lambda$ is not an eigenvalue.

For the sequel, label the points of the essential range of $\sigma^{\prime}(\tilde{w}(x))$ in increasing order: $s_{1}<s_{2}<\ldots<s_{N}$. (In Theorem 4.1, we may assume $\sigma_{0}=s_{1}$.) Consider the imaginary part of (4.1),

$$
\int_{0}^{1} \operatorname{Im} \beta\left(\lambda, \sigma^{\prime}(w(x))\right)|p(x)|^{2} d x=0 .
$$

Clearly, if $\lambda$ is an eigenvalue, $\operatorname{Im} \beta$ must be zero or change sign as $x$ varies. Compute

$$
\operatorname{Im} \beta(\lambda, s)=\operatorname{Im} \lambda^{2}(\bar{\lambda}-s) /|\lambda-s|^{2}=\frac{(\operatorname{Im} \lambda)}{|\lambda-s|^{2}}\left(|\lambda|^{2}-2 s \operatorname{Re} \lambda\right) .
$$

Since $\sigma^{\prime}(\tilde{w}(x)) \geqq s_{1}>0$ a.e. it is immediate that if $\operatorname{Re} \lambda \leqq 0$ with $\operatorname{Im} \lambda \neq 0$, then $\lambda$ is not an eigenvalue. But furthermore, we find that if $\operatorname{Im} \lambda \neq 0$ and $\lambda$ is an eigenvalue with $\operatorname{Re} \lambda>0$, then

$$
\left|\lambda-s_{1}\right| \geqq s_{1} \quad \text { and } \quad\left|\lambda-s_{N}\right| \leqq s_{N} .
$$

(Square these inequalities and expand, recalling that $s_{1} \leqq \sigma^{\prime}(\tilde{w}(x)) \leqq s_{N}$ a.e.) Therefore, nonreal eigenvalues are confined to a compact set of the positive half plane which lies between two nested circles (see Figure 4).

We now consider the real part of (4.1). Actually, it is important to consider a mixture of real and imaginary parts: Whenever $-\pi / 2 \leqq \theta \leqq \pi / 2$, we find

$$
0 \geqq \operatorname{Re}\left[e^{i \theta} \int_{0}^{1}\left(\beta_{0}-\beta\left(\lambda, \sigma^{\prime}(\tilde{w})\right)\right)|p|^{2} d x\right] \text {. }
$$




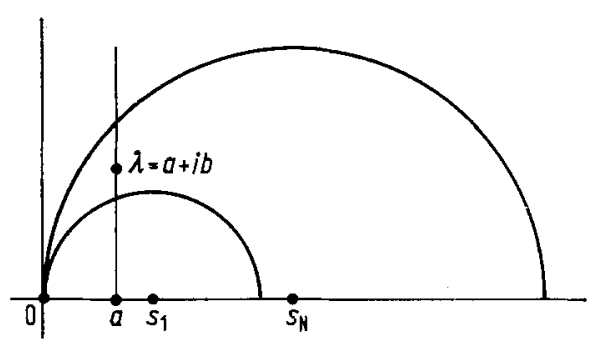

Fig. 4. $\lambda$-plane

We shall show that for each $\lambda$ with $0<\operatorname{Re} \lambda<\min \left\{s_{1}, \beta_{0} / 2\right\}$, there is a choice of $\theta$ in $[-\pi / 2, \pi / 2]$ such that

$$
\operatorname{Re}\left[e^{i \theta}\left(\beta_{0}-\beta\left(\lambda, s_{j}\right)\right]>0 \quad \text { for } j=1, \ldots, N .\right.
$$

Geometrically, this simply means that:

For $0<\operatorname{Re} \lambda<\min \left\{s_{1}, \beta_{0} / 2\right\}$, there is some line through the point

(4.2) $\beta_{0}=\pi^{2} / 4$ in the complex plane, such that the $N+1$ numbers 0 , $\beta\left(\lambda, s_{1}\right), \ldots, \beta\left(\lambda, s_{N}\right)$ lie all on one side of that line.

This then, will force $p=0$, so such $\lambda$ cannot be eigenvalues, and will finish the proof of the theorem. (We shall see that the line may not always be chosen vertical in (4.2), corresponding to $\theta=0$ above.)

To establish (4.2), fix $\lambda=a+i b$ with $0<a<\min \left\{s_{1}, \beta_{0} / 2\right\}$ and for convenience take $b>0$. We will consider the curves $\beta\left(a+i t, s_{j}\right)$ for $t \geqq 0$, $j=1, \ldots, N$, describing their shape and the relative positions along these curves of the points $\beta\left(a+i b, s_{j}\right)$.

In particular, I claim:

(1) With $t^{*}(s)=\sqrt{a(2 s-a)}$ (for any $\left.s>a\right)$ one has

$$
\begin{gathered}
\operatorname{Im} \beta(a+i t, s) \leqq 0 \quad \text { for } 0 \leqq t \leqq t^{*}(s), \\
\operatorname{Im} \beta(a+i t, s)>0 \quad \text { for } t>t^{*}(s) \\
\beta\left(a+i t^{*}(s), s\right)=2 a, \quad \beta(a, s)=\frac{-a^{2}}{s-a}<0 .
\end{gathered}
$$

(2) The curve $\beta(a+i t, s)$, for $s>a$ fixed, is convex to the left that is,

$$
\arg \frac{d \beta}{d t}=\arg i \frac{\partial \beta}{\partial \lambda}(a+i t, s)
$$

is a strictly increasing function of $t$ for $t \geqq 0$, with

$$
\left.\arg \frac{d \beta}{d t}\right|_{t=0}=-\pi / 2<\arg \frac{d \beta}{d t}<\pi / 2 \text { for all } t>0 .
$$

(Hence $\operatorname{Re} \beta(a+i t, s)$ is increasing in $t$, so $\operatorname{Re} \beta<2 a$ (respectively $>2 a$ ) if $t<t^{*}(s)$ (respectively $>t^{*}(s)$ ).) 
(3) If $a<s_{i}<s_{j}$, the curves $\beta(a+i t, s) \quad s=s_{i}, s_{j}$, are nested as follows: If

then

$$
\operatorname{Re} \beta\left(a+i t_{1}, s_{i}\right)=\operatorname{Re} \beta\left(a+i t_{2}, s_{j}\right)<2 a,
$$

$$
\operatorname{Im} \beta\left(a+i t_{1}, s_{i}\right)<\operatorname{Im} \beta\left(a+i t_{2}, s_{j}\right)<0 .
$$

(That is, the first curve lis below the second, for $\operatorname{Re} \beta<2 a$.) If

then

$$
\operatorname{Re} \beta\left(a+i t_{1}, s_{i}\right)=\operatorname{Re} \beta\left(a+i t_{2}, s_{j}\right)>2 a,
$$

$$
\operatorname{Im} \beta\left(a+i t_{1}, s_{l}\right)>\operatorname{Im} \beta\left(a+i t_{2}, s_{j}\right)>0
$$

(so the first curve lies above the second for $\operatorname{Re} \beta>2 a$ ).

Assuming these claims hold, we establish (4.2). Since $t^{*}(s)$ is monotonically increasing in $s$, for each $t>0$ there exists $j, 1 \leqq j \leqq N$, so that

$$
\begin{array}{ll}
\operatorname{Im} \beta\left(a+i t, s_{k}\right) \geqq 0 & \text { if } k \leqq j, \\
\operatorname{Im} \beta\left(a+i t, s_{k}\right)<0 & \text { if } k>j .
\end{array}
$$

Now the convexity and nesting properties (2) and (3) imply that the tangent line to the curve $\beta\left(a+i t, s_{j}\right)$ at $t=t^{*}\left(s_{j}\right)$ (where $\beta=2 a$ ) is a line with the property we desire: the points $0, \beta\left(a+i t, s_{k}\right), k=1, \ldots, N$ lie all above this line (which has positive slope), and the point $\beta_{0}>2 a$ lies below (see Figure 5). (4.2) follows.

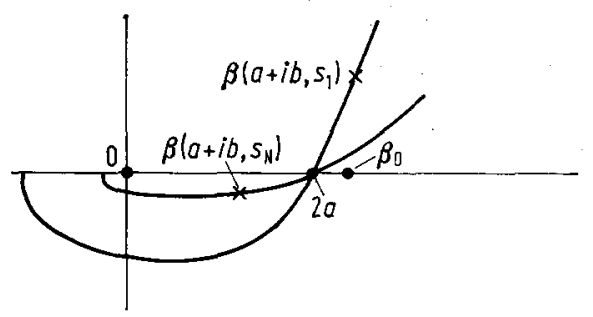

Fig. 5. $\beta\left(\lambda, s_{j}\right)$ plane

Remark. If in fact $\beta_{0}=2 a$ but $\beta\left(\lambda, s_{k}\right)+\beta_{0}$ for all $k$, the argument above shows again $\lambda$ is not an eigenvalue. Then a neighborhood of such a $\lambda$ must be in the resolvent set of the operator $A \rightarrow B$.

It remains to establish the claims (1), (2), (3). Recall that when $\operatorname{Im} \lambda>0$, $\operatorname{sgn} \operatorname{Im} \beta(\lambda, s)=\operatorname{sgn}\left(|\lambda|^{2}-2 s \operatorname{Re} \lambda\right)$.

For $\lambda=a+i t$, this equals $\operatorname{sgn}\left(a(a-2 s)+t^{2}\right)$, which is positive or negative as $t$ is greater or less than $t^{*}(s)$. When $t=t^{*}(s)$,

$$
\begin{aligned}
\operatorname{Re} \beta(\lambda, s)=\operatorname{Re} \frac{\lambda^{2}(\bar{\lambda}-s)}{|\lambda-s|^{2}} & =\frac{\left|\lambda^{2}\right| \operatorname{Re} \lambda-s\left((\operatorname{Re} \lambda)^{2}-(\operatorname{Im} \lambda)^{2}\right)}{|\lambda-s|^{2}} \\
& =\frac{\operatorname{Re} \lambda\left(|\lambda|^{2}-2 s \operatorname{Re} \lambda\right)+s|\lambda|^{2}}{|\lambda-s|^{2}}=\frac{s\left(a^{2}+t^{2}\right)}{(a-s)^{2}+t^{2}}=2 a .
\end{aligned}
$$


To check the convexity property (2), compute

$$
\frac{\partial \beta}{\partial \lambda}(\lambda, s)=\frac{\lambda(\lambda-2 s)}{(\lambda-s)^{2}}
$$

Now

$$
\begin{aligned}
\arg \frac{d \beta}{d t}(a+i t, s) & =\arg i \frac{\partial \beta}{\partial \lambda}=\frac{\pi}{2}+\arg \lambda-2 \arg (\lambda-s)+\arg (\lambda-2 s) \\
& =-\frac{\pi}{2}+\tan ^{-1}(t / a)+2 \tan ^{-1}\left(\frac{t}{s-a}\right)-\tan ^{-1}\left(\frac{t}{2 s-a}\right) .
\end{aligned}
$$

Then

$$
\begin{aligned}
\frac{d}{d t}\left(\arg \frac{d \beta}{d t}(a+i t, s)\right) & =\frac{a}{a^{2}+t^{2}}+\frac{2(s-a)}{(s-a)^{2}+t^{2}}-\frac{(2 s-a)}{(2 s-a)^{2}+t^{2}} \\
& >\frac{a+2(s-a)-(2 s-a)}{(2 s-a)^{2}+t^{2}}=0
\end{aligned}
$$

if $s>a$. Since clearly $\lim _{t \rightarrow \infty} \arg \frac{d \beta}{d t}(a+i t, s)=\pi / 2$, the fact that $-\pi / 2 \leqq$ $\arg \frac{d \beta}{d t}(a+i t, s)<\pi / 2$ follows.

To verify the nesting property (3), it is enough to show that the direction of $\frac{\partial \beta}{\partial s}(a+i t, s)$ is to the left (respectively right) of the tangent $\frac{d \beta}{d t}=i \frac{\partial \beta}{\partial \lambda}(a+i t, s)$, whenever $\operatorname{Re} \beta<2 a$ (respectively $>2 a$ ). This follows from the fact

$$
\operatorname{sgn} \operatorname{Re} \frac{\partial \beta}{\partial \lambda} \frac{\partial \beta}{\partial s}(a+i t, s)=\operatorname{sgn} \operatorname{Im} \beta(a+i t, s)
$$

which we now check:

$$
\frac{\partial \beta}{\partial \lambda} \frac{\overline{\partial \beta}}{\partial s}=\frac{\lambda(\lambda-2 s)}{(\lambda-s)^{2}} \overline{\left(\frac{\lambda}{\lambda-s}\right)^{2}}=\frac{|\lambda|^{2}\left(|\lambda|^{2}-2 s \bar{\lambda}\right)}{|\lambda-s|^{4}} .
$$

Thus sgn $\operatorname{Re} \frac{\partial \beta}{\partial \lambda} \frac{\overline{\partial \beta}}{\partial s}=\operatorname{sgn}\left(|\lambda|^{2}-2 s \operatorname{Re} \lambda\right)=\operatorname{sgn} \operatorname{Im} \beta$. This finishes the proof of Theorem 4.1.

We conclude this section with a criterion for instability:

Remark 4.2. If in 4.1 the equilibrium solution $(0, \tilde{w}(x))$ satisfies, instead of (2.4), (4.3) $\sigma(\tilde{w}(x))=P$ a.e., $\sigma^{\prime}(\tilde{w}(x))<0$ for $x$ in a set of positive measure in $(0,1)$ then $\tilde{z}(x)=(0, \tilde{w}(x))$ is unstable in the sense that there exists $\varepsilon_{0}>0$ and a sequence of initial data $z_{0}^{n} \rightarrow \tilde{z}$ in $X^{\frac{1}{2}}$ so that if $z_{n}(t)$ denotes the solution of (3.1) 
with initial data $z_{0}^{n}$ for all $n$ we have

$$
\sup _{t \geq 0}\left\|z_{n}(t)-\tilde{z}\right\|_{X^{\frac{1}{2}}} \geqq \varepsilon_{0}>0
$$

the supremum taken over the maximal interval of existence of $z_{n}(t)$. This fact is an application of the statements of HENRY 5.1.3, the point being that if (4.3) holds, then the essential spectrum of $A-B$ intersects the unstable half plane $\{\operatorname{Re} \lambda<0\}$, since the essential spectrum is the essential range of $\sigma^{\prime}(\tilde{w}(x))$ as demonstrated in the proof of 4.1 .

\section{Global Existence and Convergence to Equilibrium}

The method we use to establish global existence of the solution of (2.9) is the same as that of ANDREwS \& BALL; we include a dicussion for completeness. In this method, the sign of the stress for extreme ranges of strain is restricted in a reasonable way, and also the energy of the initial data is restricted (see 5.2 below). However, if $\sigma(w)$ is defined for all $w \in \mathbb{R}$, and satisfies, for some $M>0$,

$$
w(\sigma(w)-P)>0 \quad \text { if }|w|>M,
$$

then the energy of the initial data is unrestricted.

As we begin, let us first establish the energy identity:

Proposition 5.1. Assume $\sigma(w)$ is locally Lipschitz-continuous. Suppose a solution $(p, q)$ to problem $(2.9)$ exists for $0 \leqq t \leqq T$ with $p_{0} \in W^{1,2}, p_{0}(1)=0$, and $q_{0} \in L^{\infty}$. Then the energy identity (2.13) holds and each term is continuous in $t$ for $t \geqq 0$.

Proof. Multiply equation $\left(2.9_{1}\right)$ for $p$ by $p_{x x}=(p+q)_{t}$ and integrate over $x$. Using the regularity of the solution as established in Theorem 3.1 and the boundary conditions in $\left(2.9_{3}\right)$, we find that for $t>0$,

and

$$
\int_{0}^{1} p_{x x} p_{t}(x, t) d x=-\int_{0}^{1} p_{x} p_{x t}(x, t) d x=-\left(\int_{0}^{1} \frac{1}{2} p_{x}^{2}(x, t) d x\right)_{t}
$$

$$
\int_{0}^{1}(\sigma(p+q)-P)(p+q)_{t}(x, t) d x=\left(\int_{0}^{1}(W(p+q)-P(p+q))(x, t) d x\right)_{t}
$$

We integrate from $t=\varepsilon>0$ to $T$ and let $\varepsilon \rightarrow 0$; then (2.14) follows.

In the global existence theorem below, we fix a compact interval $\left[w_{-}, w_{+}\right] \subset J$ (the domain of $\sigma$ ), and fix $M, 0<M<\left(w_{+}-w_{-}\right) / 2$, so that also $\left[w_{-}-M\right.$, $\left.w_{+}+M\right] \subset J$. We require that $\sigma(w)$ satisfy

$$
\begin{array}{ll}
\sigma(w)-P<0 & \text { if }\left|w-w_{-}\right|<M \\
\sigma(w)-P>0 & \text { if }\left|w-w_{+}\right|<M .
\end{array}
$$

Adjusting the stored energy function $W(w)$ by a constant if necessary, we may assume $\min \left\{W(w)-P w \mid w \in\left[w_{-}-M, w_{+}+M\right]\right\}=0$. 
Theorem 5.2 (Global existence). Assume $\sigma(w)$ is locally Lipschitz-continuous. Suppose the initial data for (2.9) satisfy $p_{0} \in W^{1,2}, p_{0}(1)=0, q_{0} \in L^{\infty}$, and

$$
\begin{gathered}
q_{0}(x) \in\left[w_{-}, w_{+}\right] \text {a.e. } \\
\int_{0}^{1}\left(\frac{1}{2} p_{0 x}^{2}+W\left(p_{0}+q_{0}\right)\right)(x) d x<M^{2} / 2 .
\end{gathered}
$$

Then the solution $(p, q)$ of the problem (2.9) exists globally for $t \geqq 0$ and satisfies, for all $t \geqq 0$,

$$
\begin{array}{cc}
|p(x, t)|<M & \text { for all } x \text { in }(0,1) \\
q(x, t) \in\left[w_{-}, w_{+}\right] & \text {for a.e. } x \text { in }(0,1) .
\end{array}
$$

Corollary 5.3. (Classical solutions). Suppose that $\sigma(w)$ is $C^{1}$ and $\sigma^{\prime}(w)$ is locally Lipschitz-continuous. In addition to the hypotheses of Theorem 5.2, assume $p_{0} \in$ $W^{2,2}(0,1), p_{0 x}(0)=0$, and $q_{0} \in C^{1}[0,1]$. Then $q(x, t)$ is $C^{1}$ in $(x, t)$ for all $t \geqq 0$, so that the solution $u(x, t)$ of $(2.3)$ obtained from $(p, q)$ remains classical for all $t>0$. below.

The proof of the corollary will be postponed until after the proof of Theorem 5.4

Proof of 5.2. Assume the solution exists for $0 \leqq t<T$ and $T$ is maximal. We claim that the bounds (5.4) along with the inequality $W(p+q)-P(p+q) \geqq 0$ a.e. hold for $0 \leqq t<T$. In particular we will show that the set of $t_{0}$ such that (5.4) and (5.5) hold for $0 \leqq t \leqq t_{0}$ is open and closed in [0 T). Suppose (5.4) holds for $0 \leqq t \leqq t_{0}$. Since $p(x, t)$ is continuous, there is $\varepsilon>0$ so $|p(x, t)|$ $<M+\varepsilon, 0<x<1,0 \leqq t \leqq t_{0}$. Then since $q$ is continuous into $L^{\infty}$, there is $\delta>0$ so $(p+q)(x, t) \in\left(w_{-}-M, w_{+}+M\right)$ for $0 \leqq t \leqq t_{0}+\delta$, and a.e. $x$. This implies that $W(p+q)-P(p+q) \geqq 0$ for a.e. $x, 0 \leqq t \leqq$ $t_{0}+\delta$, and the energy identity (2.13) now yields

$$
|p(x, t)| \leqq\left\|p_{x}(t)\right\|_{L^{2}}<M \quad \text { for } 0 \leqq x \leqq 1,0 \leqq t \leqq t_{0}+\delta .
$$

Using Corollary 3.5, for each $x$ in a set of full measure we may apply Lemma 1.1 to the function $q(t)=q(x, t), x$ fixed, because of the hypothesis (5.2), and conclude that the interval $\left[w_{-}, w_{+}\right]$is invariant for the ordinary differential equation $\left(2.9_{2}\right)$ for $0 \leqq t \leqq t_{0}+\delta$. Thus (5.4) holds for $0 \leqq t \leqq t_{0}+\delta$.

It is easy to verify that if (5.4) holds for $0 \leqq t<t_{0}<T$ then it holds for $0 \leqq t \leqq t_{0}$. Thus we have shown that (5.4) holds for $0 \leqq t<T$. We may now apply a continuation theorem of HENRY 3.3 .4 , to conclude that $T=+\infty$, because (5.4) implies that $\{z(t) \mid z(t)=(p, q)(t), 0<t<T\}$ is contained in a closed, bounded subset of the domain of definition of $f(z)=(\sigma(p+q)-P$, $-\sigma(p+q)+P)$ in $X^{\frac{1}{2}}=\left(W_{b}^{1,2}, L^{\infty}\right)$, on which $f$ is bounded in $X=\left(L^{2}, L^{\infty}\right)$. This completes the proof of 5.2. We note that we have shown that the solution satisfies, for some $M_{1}>0$,

$$
\|\sigma(p+q)(t)-P\|_{L} \infty \leqq M_{1} .
$$


The main result in this section is:

Theorem 5.4 (Approach to equilibrium). Under the hypotheses of Theorem 5.2, the solution $(p, q)$ of (2.9) has the following asymptotic behavior as $t \rightarrow \infty$ :

$$
\|p(t)\|_{W^{2,2}} \rightarrow 0
$$

$q(x, t) \rightarrow w^{\infty}(x) \quad$ boundedly a.e.,

$$
\text { where } \quad w^{\infty} \in L^{\infty} \text { and } \sigma\left(w^{\infty}(x)\right)=P \text { a.e. }
$$

$$
\begin{gathered}
\sigma(p+q)(x, t) \rightarrow P \quad \text { boundedly a.e. } \\
\|S(t)\|_{C^{1, v}} \rightarrow 0 \quad \text { for any } v<1 / 2
\end{gathered}
$$

If $\sigma(w)$ is $C^{1}$ with $\sigma^{\prime}(w)$ locally Lipschitz-continuous, then also

$$
\|S(t)\|_{W^{2,2}} \rightarrow 0
$$

We shall see in $\S 6$ that in general, one cannot expect $\sigma$, the elastic part of the stress, to approach $P$ uniformly in $x$. We note that in terms of the solution $u(x, t)$ of (2.3) obtained from $(p, q)$, the results of the theorem above yield

$$
\begin{gathered}
\left\|u_{t}(t)\right\|_{W^{1,2}} \rightarrow 0 \\
u_{x}(x, t) \rightarrow w^{\infty}(x) \quad \text { boundedly a.e. }
\end{gathered}
$$

Proof. The first step is to establish that

$$
\|p(t)\|_{W^{1,2}} \rightarrow 0 \quad \text { as } t \rightarrow \infty .
$$

From the Poincaré inequality, using the boundary condition $p_{x}(0, t)=0$, we have

$$
\int_{0}^{\infty} \int_{0}^{1} p_{x}^{2}(x, t) d x d t<\int_{0}^{\infty} \int_{0}^{1} p_{x x}^{2}(x, t) d x d t \leqq M^{2} / 2
$$

this last following from the energy identity. I claim that $\left\|p_{x}(t)\right\|_{L^{2}}^{2}$ has a bounded continuous time derivative on $[\delta, \infty)$ for any $\delta>0$, so that $(5.7)$ follows. That such is the case follows from Lemma A.3 in the Appendix, an abstract statement of smoothing which sharpens a conclusion of HENRY 3.5.2 to achieve some explicit bounds: For the abstract equation (3.1), it follows from A.3 and the proof of 3.1 that $z_{t} \in C\left((0, \infty), X^{\frac{1}{2}}\right)$, with

$$
\left\|z_{t}(t)\right\|_{X^{\frac{1}{2}}} \leqq C_{*}(T)\left(t-t_{0}\right)^{-1}\left(\left\|z\left(t_{0}\right)\right\|_{X^{\frac{1}{2}}}+\sup _{t_{0} \leqq s \leqq t}\|f(z(s))\|_{X}\right)
$$

for any $t, t_{0}$ with $0<t-t_{0}<T$ ( $T$ fixed). Taking $t-t_{0}=1$ and using the established bounds (5.4), (5.5), and (5.6) to get bounds for $z(t)$ and $f(z(t))$, we conclude that for some $M_{2}>0$,

$$
\left\|p_{t}(t)\right\|_{W^{1,2}} \leqq M_{2} \quad \text { for all } t \geqq 1 .
$$

This yields the desired result (5.7), put also has some independent interest since $p_{t}=S$, the total stress. 
Step two in the proof (perhaps the most significant) is to reconsider the ordinary differential equation $\left(2.9_{2}\right), q_{t}=-\sigma(p+q)+P$. Since from (5.7), $\|p(t)\|_{L^{\infty}} \rightarrow 0$ as $t \rightarrow \infty$, this ordinary differential equation is "asymptotically autonomous." A simple lemma describes the asymptotic behavior of its solutions:

Lemma 5.5. Suppose $q(t)$ is $C^{1}$ for $t \in(0, \infty)$, with $|q(t)| \leqq C$ for all $t, p(t)$ is continuous with $p(t) \rightarrow 0$ as $t \rightarrow \infty$, and $q_{t}=-\sigma(p+q)+P$. Then there is a constant $q^{\infty}$ such that $q(t) \rightarrow q^{\infty}$ as $t \rightarrow \infty$, and $\sigma\left(q^{\infty}\right)=P$.

I thank J. BALL for the proof below, which is simpler and more general than the original proof:

Let $\underline{q}=\lim _{t \rightarrow \infty} q(t), \bar{q}=\varlimsup_{t \rightarrow \infty} q(t)$. Supposing $\underline{q}<\bar{q}$, choose any $\left.q_{0} \in \underline{(q}, \bar{q}\right)$. Then there exist two sequences $t_{i}^{ \pm} \rightarrow \infty$ such that $q\left(t_{i}^{ \pm}\right)=q_{0}$ and $q_{t}\left(t_{i}^{+}\right) \geqq 0$ $\geqq q_{t}\left(t_{i}^{-}\right)$for all $i$. Since $q_{t}\left(t_{i}^{ \pm}\right)=-\sigma\left(q_{0}+p\left(t_{i}^{ \pm}\right)\right)+P$, letting $i \rightarrow \infty$ we find $\sigma\left(q_{0}\right)=P$. Thus $\sigma \equiv P$ on $[q, \bar{q}]$, since $q_{0}$ was arbitrary. But now if $T$ is so large that $|p(t)|<\varepsilon(\vec{q}-\underline{q})$ for $\bar{t}>T$, we have $q_{t}(t)=0$ whenever $q \in(\underline{q}+\varepsilon$, $\bar{q}-\varepsilon$ ). This is not possible if $\underline{q}<\bar{q}$. Hence $\underline{q}=\bar{q}=\lim _{t \rightarrow \infty} q(t)$.

Recall that for $x$ is a set of full measure in $(0,1)$ the ordinary differential equation $\left(2.9_{2}\right)$ is satisfied classically for $t>0$. With $x$ fixed, the lemma above applies to $q(t)=q(x, t)$, and we conclude that as $t \rightarrow \infty, q(x, t) \rightarrow q^{\infty}(x)$ boundedly almost everywhere pointwise in $x$. In particular, $q^{\infty} \in L^{\infty}(0,1)$, and $\sigma\left(q^{\infty}(x)\right)=P$ a.e. We also conclude that $\sigma(p+q)(x, t) \rightarrow P$ as $t \rightarrow \infty$ boundedly a.e., so $\sigma \rightarrow P$ in $L^{2}$ and $q_{t} \rightarrow 0$ in $L^{2}$ as $t \rightarrow \infty$.

The next step is to show that as $t \rightarrow \infty$

$$
\left\|p_{t}\right\|_{C^{1, \nu}} \rightarrow 0 \text { for any } v<\frac{1}{2} .
$$

From equation (2.9 ) it then follows that $p_{x x}=p_{t}-\sigma+P \rightarrow 0$ in $L^{2}$ (but not in $L^{\infty}$, see $\S 6$ ).

To establish (5.9) we shall regard $\left(2.9_{1}\right)$ as an abstract equation

$$
p_{t}=\Delta p+g(p, t)
$$

in the space $Y=L^{2}$, with $\Delta$ and $D(\Delta)$ as in the proof of 3.1, and with $g(p, t)=$ $\sigma(p+q(t))-P$. As in $\S 3, \quad Y^{v}=\left(D(-\Delta)^{\gamma}\right) C C^{1, \nu} \quad$ if $\quad 2 \gamma>v+3 / 2 \quad$ and $Y^{\frac{1}{2}}=W_{b}^{1,2}$. Observing that

$$
\|q(t)-q(s)\|_{L^{2}} \leqq K(s)(t-s)
$$

for $t>s>0$ where $K(s)=\sup _{s<t<\infty}\left\|q_{t}(t)\right\|_{L^{2}} \rightarrow 0$ as $s \rightarrow \infty$, we have

$$
\|g(p(t), t)-g(p(s), s)\|_{L^{2}} \leqq L\left(\|p(t)-p(s)\|_{L^{2}}+K\left(t_{0}\right)(t-s)\right)
$$

whenever $t_{0}<s<t$ (where $L$ depends on the bounds in (5.4)). Applying Lemma A.3 in this context, we find, for $0<t_{0}<t<t_{0}+T$ ( $T$ fixed), and any 
$\gamma<1$

$$
\left\|p_{t}(t)\right\|_{Y^{\gamma}} \leqq C_{*}(T)\left(t-t_{0}\right)^{-\gamma-\frac{1}{2}}\left(\left\|p\left(t_{0}\right)\right\|_{Y}+\sup _{t_{0} \leqq t \leqq T+t_{0}}\|g(p(t), t)\|_{Y}+K\left(t_{0}\right)\right) .
$$

Fixing $t-t_{0}=1$ and letting $t_{0} \rightarrow \infty$, we derive (5.9).

The last goal is to show that $p_{t x x}=S_{x x} \rightarrow 0$ in $L^{2}$ as $t \rightarrow \infty$, provided $\sigma(w)$ is $C^{1}$ and $\sigma^{\prime}(w)$ is locally Lipschitz-continuous. Since $S$ is a strong solution of

$$
S_{t}=S_{x x}+\sigma^{\prime}(p+q)(S-\sigma(p+q)+P)
$$

for $t>0(c f . \S 3)$, it suffices to show that

$$
\left\|S_{t}(t)\right\|_{L^{2}} \rightarrow 0 \quad \text { as } t \rightarrow \infty .
$$

But the proof of this goes the same as the proof of (5.9). This completes the proof of Theorem 5.4.

Proof of 5.3. As in the proof of 5.2, we need to show that if $T$ is finite, the set $\{z(t) \mid z(t)=(p, q)(t), 0<t<T\}$ is contained in a closed, bounded subset of the domain of definition of $f(z)$ in $X^{\alpha}=\left(D\left((-A)^{\alpha}\right), C^{1}\right) \quad(3 / 4<\alpha<1$, as in the proof of 3.6) on which $f$ is bounded in $X=\left(L^{2}, C^{1}\right)$. Beyond the argument in the proof of 5.2, it suffices to show that the quantities $\left\|p_{x x}\right\|_{L^{2}}$ and $\left\|q_{x}\right\|_{L^{2}}$ cannot blow up in finite time.

Now $p_{x x}=p_{t}-\sigma(p+q)+P$, so in fact $\left\|p_{x x}\right\|_{L^{2}} \leqq M_{1}+M_{2}$ for $t \geqq 1$ by (5.6) and (5.8). On the other hand, $q_{x}$ satisfies a linear ordinary differential equation,

$$
q_{x t}=-\sigma^{\prime}(p+q)\left(q_{x}+p_{x}\right),
$$

with bounded coefficients and forcing so $q_{x}$ grows at an exponential rate at worst.

Q.E.D.

\section{Discontinuous Asymptotic States for Smooth Solutions}

Each solution constructed in $\S 5$ approaches some unique stationary asymptotic state as $t \rightarrow \infty$. The conclusion of $\S 4$ about stability identifies the asymptotic state when the initial data lie close to a stationary state which satisfies (2.4). But if the stationary state has discontinuous strain $\tilde{w}(x)$, then Theorem 4.1 applies only if the initial strain is close to the stationary strain in $L^{\infty}$ and hence is discontinuous itself. On the other hand, smooth initial data yield classical solutions with continuous strain for all $t$ (DAFERMos (1969) and Theorem 3.6 above). What happens if the initial data are smooth, but approximate, except in some thin transition layers, some stationary state satisfying (2.4) but having discontinuous strain? The results below show that one can guarantee in this situation that the asymptotic state has discontinuous strain which is equal to the unperturbed strain except on a small set. Essentially, we show that stationary states satisfying (2.4) are stable to perturbations with small energy, in a sense. 
Fix an equilibrium state $(0, \tilde{w}(x))$ for $(2.9)$, so $\sigma(\tilde{w}(x))=P$ a.e. Suppose at first that $\sigma(w)$ is $C^{1}$, and that $\sigma^{\prime}(\tilde{w}(x)) \geqq \sigma_{0}>0$, i.e. (2.4) holds. Then the essential range of $\tilde{w}(x)$ must be a finite set $\left\{w_{1}, \ldots, w_{N}\right\}$. We assume $w_{1}<w_{2}<\ldots$ $\ldots<w_{N}$. We may choose intervals $B_{j}=\left(w_{j}^{-}, w_{j}^{+}\right)$and $E>0$ such that

$$
\begin{gathered}
E<\min \left\{\left|w_{j}-w_{j}^{ \pm}\right|\right\}, \\
w_{j} \in\left(w_{j}^{-}, w_{j}^{+}\right) \quad \text { for } j=1, \ldots, N, \\
\sigma(w)<P \quad \text { if } w_{j}^{-}-E<w<w_{j}, \\
\sigma(w)>P \quad \text { if } w_{j}<w<w_{j}^{+}+E .
\end{gathered}
$$

In the theorem below, we assume only that $\sigma(w)$ is locally Lipschitz-continuous, but require that the essential range of $\tilde{w}(x)$ be a finite set as above and that (6.1) holds. We note that by Lemma 2.1, provided that $|p(x, t)|<E$ for all $(x, t)$, the intervals $B_{j}$ are each positively invariant for the ordinary differential equation $\left(2.9_{2}\right)$. Below, the Lesbegue measure of a set $S$ is denoted by $\mu(S)$.

Theorem 6.1 (Stability of equilibria). Assume that the initial data $u_{0} \in L^{\infty}, u_{1} \in L^{2}$ for the problem (2.3) satisfy, with $q_{0}(x)=u_{0}(x)-\int_{1}^{x} u_{1}(y) d y$,

and

$$
\begin{gathered}
q_{0}(x) \in\left[w_{1}^{-}, w_{N_{i}}^{+}\right] \text {a.e., } \\
\int_{0}^{1} \frac{1}{2} u_{1}^{2}(x) d x+\int_{0}^{1}\left(W\left(u_{0}(x)\right)-P u_{0}(x)\right) d x \\
<\frac{E^{2}}{2}+\int_{0}^{1} W_{m}\left(q_{0}(x)\right) d x
\end{gathered}
$$

$$
\begin{gathered}
\text { either } \sup _{0 \leqq x \leqq 1}\left|\int_{1}^{x} u_{1}(y) d y\right|<E \\
\text { or } \int_{0}^{1}\left(W\left(u_{0}(x)\right)-P u_{0}(x)\right) d x \geqq \int_{0}^{1} W_{m}\left(q_{0}(x)\right) d x,
\end{gathered}
$$

where $W^{\prime}(w)=\sigma(w), \quad \inf \left\{W(w)-P w \mid w \in\left[w_{1}^{-}-E, w_{N}^{+}+E\right]\right\}=0$, and

$$
W_{m}(q)=\left\{\begin{array}{cl}
W\left(w_{j}\right)-P w & \text { if } q \in B_{j} \text { for some } j \\
0 & \text { if } q \notin B_{j} \text { for all } j .
\end{array}\right.
$$

Then the solution $u(x, t)$ of (2.3) exists for all $t>0$, and $u_{x}(x, t)$ converges boundedly a.e. as $t \rightarrow \infty$ to an equilibrium state $w^{\infty}(x)$ with $\sigma\left(w^{\infty}(x)\right)=P$ a.e., and in particular,

$$
w^{\infty}(x)=w_{j} \quad \text { if } q_{0}(x) \in B_{j} \text { for a.e. } x
$$

Thus, if

$$
\mu\left(\left\{x|| q_{0}(x)-\tilde{w}(x) \mid>E\right\}\right)<\varepsilon,
$$

then

$$
\mu\left(\left\{x \mid w^{\infty}(x) \neq \tilde{w}(x)\right\}\right)<\varepsilon .
$$


This theorem identifies a certain "basin of stability" for a stationary state satisfying (6.1) and achieves pointwise control over the asymptotic state of solutions in (6.5). I emphasize that this basin contains smooth data $\left(u_{0}, u_{1}\right)$ which satisfies (6.3), (6.4), and (6.6), for any positive $E$ and $\varepsilon$. In particular, given a sequence $\left\{\left(u_{0}^{n}, u_{1}^{n}\right)\right\}$ with $u_{1}^{n} \rightarrow 0$ in $L^{2}, u_{0}^{n} \rightarrow \tilde{w}(x)$ boundedly a.e., then (6.3), (6.4) and (6.6) must hold for $n$ sufficiently large. If the stationary strain $\tilde{w}(x)$ is not constant, and the initial strain $u_{0}(x)$ is smooth and satisfies (6.6) for $\varepsilon$ sufficiently small, then $q_{0}(x)$ is close to $\tilde{w}(x)$ except in some "transition layers" of small measure. Then (6.5) identifies the asymptotic strain $w^{\infty}(x)$ pointwise from the initial data except in these transition layers, and in particular $w^{\infty}(x)$ must be nonconstant and discontinuous, since $\sigma\left(w^{\infty}(x)\right)=P$ a.e. Since smooth initial data yield classical solutions with $C^{1}$ strain, convergence of the strain as $t \rightarrow \infty$ does not occur uniformly. We note that the behavior of the strain in the transition layers which must persist is governed by the ordinary differential equation $\left(2.9_{2}\right)$.

Proof of 6.1. We work with the corresponding solution $(p, q)$ of the system (2.9). Recall $u_{t}=p_{x}, u_{x}=p+q$, and the energy identity (2.13). I claim that the solution $(p, q)$ exists globally in $t$, satisfying for $t>0$

$$
\begin{gathered}
|p(x, t)| \leqq\left\|p_{x}^{*}(t)\right\|_{L^{2}}<E \quad \text { for } 0 \leqq x \leqq 1 \\
q(x, t) \in\left[w_{1}^{-}, w_{N}^{+}\right] \quad \text { for a.e. } x \\
(W(p+q)-P(p+q))(x, t) \geqq W_{m}\left(q_{0}(x)\right) \quad \text { for a.e. } x
\end{gathered}
$$

As in the proof of Theorem 5.2, it suffices to establish these inequalities for $t \in[0, T)$, where $[0, T)$ is the maximal interval of existence. As before, the strategy is to show that the set of $t_{0}<T$ such that (6.8)-(6.10) hold for $0 \leqq t \leqq t_{0}$ is open and closed in $[0, T)$. It is easy to show this set is closed, using the energy identity to get (6.8). Now suppose (6.8)-(6.10) hold for $0 \leqq t \leqq t_{0}<T$. Then by continuity, (6.8) holds for $0 \leqq t \leqq t_{0}+\delta$ for some $\delta>0$. Using Lemma 2.1, this implies that the intervals $B_{j}, j=1, \ldots, N$, and the interval $\left[w_{1}^{-}, w_{N}^{+}\right]$are all positively invariant for the ordinary differential equation $\left(2.9_{2}\right)$ for $0 \leqq t$ $\leqq t_{0}+\delta$, for $x$ in a set of full measure in $[0,1]$. Thus (6.9) holds. Now certainly $W(p+q)-P(p+q) \geqq 0$ for a.e. $x$, for $0 \leqq t \leqq t_{0}+\delta$. But also, for a.e. $x$ such that $q_{0}(x) \in B_{j}$ we must then have $q(x, t) \in B_{j}$ for $0 \leqq t \leqq t_{0}+\delta$. Using (6.8) and (6.1) we find that for such $x$,

$$
\begin{gathered}
(W(p+q)-P(p+q))(x, t) \geqq W\left(w_{j}\right)-P_{w_{j}}= \\
\inf \left\{W(w)-P w \mid w \in\left[w_{j}^{-}-E, w_{j}^{+}+E\right]\right\}=W_{m}\left(q_{0}(x)\right) .
\end{gathered}
$$

Thus the lower bound (6.10) on the stored energy holds for $0 \leqq t \leqq t_{0}+\delta$ as well.

Global existence follows, and indeed the conclusions of Theorem 5.4 hold for this solution as well. We conclude that $\|p(t)\|_{W^{2,2} \rightarrow 0}$ as $t \rightarrow \infty$, and that for $x$ in a set of full measure, $q(x, t) \rightarrow w^{\infty}(x)$ as $t \rightarrow \infty$, where $\sigma\left(w^{\infty}(x)\right)=P$. Then

$$
u_{x}(x, t)=(p+q)(x, t) \rightarrow w^{\infty}(x) \quad \text { as } t \rightarrow \infty \quad \text { for a.e. } x
$$


Since the intervals $B_{j}$ are invariant for the ordinary differential equation (2.9. it follows that if $q_{0}(x) \in B_{j}$ for some $j$, then $w^{\infty}(x) \in \bar{B}_{j}$. But by $(6.1), w_{j}$ is the only solution of $\sigma(w)=P$ in $\bar{B}_{j}$, so we must have $w^{\infty}(x)=w_{j}$. This finishes the proof of Theorem 6.1.

Note. WeINBERger (1982) has described the phenomenon of an uncountable infinity of discontinuous, stable, asymptotic states for classical solutions in a population model with nearly the same structure as the system (2.9). Here, though, the special structure of (2.9) makes it possible to obtain pointwise information about the asymptotic state with the simple techniques used above.

More can be said about the ultimate structure of the transition layers if they are sufficiently steep at first. We consider classical solutions as constructed by Theorem 3.6. Suppose $\sigma(w)$ is $C^{1}$ with $\sigma^{\prime}(w)$ locally Lipschitz-continuous. Suppose (6.1) holds. Let

$$
B_{0}=\bigcup_{j=1}^{N-1}\left[w_{j}^{+}, w_{j+1}^{-}\right]=\left[w_{1}^{-}, w_{N}^{+}\right] \backslash \bigcup_{j=1}^{N} B_{j} .
$$

Suppose, what is reasonable as in Fig. 3, that $w_{j}^{ \pm}$and $E$ have been chosen such that

$$
\sigma^{\prime}(p+q)<0 \quad \text { if } \quad q \in B_{0} \quad \text { and }|p| \leqq E .
$$

Proposition 6.2. Suppose that the initial data for (2.3) satisfy $u_{0} \in C^{1}$ and $u_{1} \in W^{1,2}$ with $u_{1}(0)=0$ and (6.2), (6.3), and (6.4). Given any $C_{1}>0$, there exists $C_{2}>0$ such that if $\left\|u_{1 x}\right\|_{L^{2}}<C_{1}$, then for any fixed $x$ such that

$$
\left|q_{0 x}(x)\right| \geqq C_{2} \quad \text { and } \quad q_{0}(x) \in B_{0}
$$

it follows that

$$
\left|q_{x}(x, t)\right| \geqq C_{2} \quad \text { whenever } \quad t \geqq 0 \quad \text { and } \quad q(x, t) \in B_{0} .
$$

In particular, it follows from 6.2 and 6.1 that a single monotone transition layer $[a, b]$ where

$$
\left|q_{0 x}(x)\right| \geqq C_{2} \quad \text { for } a \leqq x \leqq b
$$

and

$$
q_{0}(a) \in B_{j}, \quad q_{0}(b) \in B_{j+1}
$$

must persist for all time, with

$$
\left|q_{x}(x, t)\right| \geqq C_{2} \quad \text { whenever } \quad a \leqq x \leqq b \quad \text { and } \quad q(x, t) \in B_{0},
$$

and

$$
q(a, t) \in B_{j}, \quad q(b, t) \in B_{j+1} \quad \text { for all } t \geqq 0 .
$$

Proof of 6.2. From the proof of 6.1 we have

$$
|p(x, t)|<E \quad \text { for all } x \in[0,1], \quad t>0 .
$$


Thus $B_{0}$ is negatively invariant: Let $t_{0}>0$ so that $q\left(x_{0}, t_{0}\right) \in B_{0}$. Then $q\left(x_{0}, t\right) \in B_{0}$ for $0 \leqq t \leqq t_{0}$. I claim there exists $C_{2}$ such that

$$
\left|p_{x}(x, t)\right| \leqq C_{2} \quad \text { for all } x \in[0,1], t>0 .
$$

Recall that a classical solution of (2.9) satisfies

$$
q_{x t}=-\sigma^{\prime}(p+q)\left(p_{x}+q_{x}\right) .
$$

From (6.11) and (6.12) it follows that the set $\left\{r|| r \mid \geqq C_{2}\right\}$ is invariant for $q_{x}\left(x_{0}, t\right)$ in (6.13) for $0 \leqq t \leqq t_{0}$. which is enough to prove 6.2.

It remains to establish the bound (6.12), given that $\left\|p_{0 x x}\right\|_{L^{2}} \leqq C_{1}$. Set $Y=L^{2}$, with $Y^{\gamma}=D\left((-\Delta)^{\gamma}\right) C C^{1, v}$ if $2 \gamma>v+3 / 2$ as in the proof of 5.4. Fixing such a $\gamma<1$, for any $\nu<1 / 2$, we may choose $C_{3}$ from $C_{1}$ so that

$$
\left\|p_{0}\right\|_{\gamma} \leqq C\left\|p_{0}\right\|_{W^{2,2}} \leqq C_{3} .
$$

From Lemma A.1, for $0 \leqq t \leqq 1$, and from the bound for $\left\|p_{x x}(t)\right\|_{L^{2}}$ implied by (5.6) and (5.8), for $t \geqq 1$, the bound (6.12) follows, establishing Proposition 6.2.

\section{Hysteresis and Creep in a Load-Deformation Experiment}

The goal in this section is to exhibit hysteresis and creep phenomena in appropriately idealized dynamic processes in a viscoelastic bar.

Let us take $\sigma(w)$ of the form indicated in Fig. 1 and fix some notation: $\sigma$ should be locally Lipschitz-continuous, defined on $\mathbb{R}$ for convenience, strictly increasing for $w<\alpha$ and $\beta<w$ with $\alpha<\beta$ and strictly decreasing for $\alpha<w<\beta$. Set $P_{\alpha}=\sigma(\alpha), P_{\beta}=\sigma(\beta)$. For $P \leqq P_{\alpha}$ denote by $w_{\alpha}(P)$ the unique $w \leqq \alpha$ satisfying $\sigma(w)=P$, and for $P \geqq P_{\beta}$ similarly define $w_{\beta}(P)$ as that $w \geqq \beta$ so $\sigma(w)=P$. Also for $P_{\beta}<P<P_{\alpha}$ set

$$
E_{\alpha}(P)=\frac{1}{2}\left(w_{0}-w_{\alpha}(P)\right), \quad E_{\beta}(P)=\frac{1}{2}\left(w_{\beta}(P)-w_{0}\right),
$$

where $w_{0}$ is the middle root of $\sigma(w)=P$. For $P<P_{\beta}$, set $E_{\alpha}(P)=\infty$, and for $P>P_{\alpha}$, set $E_{\beta}(P)=\infty$.

We will model the load-deformation experiment very simply, as a chain of initial-boundary value problems, allowing discretely imposed changes in the load $P$ and small "fluctuations" imposed on the solution itself (as one model accounting for unknown physical influences on the bar).

Select a sequence of loads $P_{0}, P_{1}, \ldots$ (nondecreasing for now), a sequence of "fluctuations" $\left(u_{0}^{j}, u_{1}^{j}\right), j=0,1, \ldots$ in $\left(L^{\infty}, L^{2}\right)$, and a sequence of times $0=t_{0}<t_{1}<\ldots$ Under suitable restrictions, we will construct global in time solutions $u^{j}(x, t)$ to the initial-boundary value problems below, for $j=0,1,2, \ldots$ :

$$
\begin{gathered}
u_{t t}^{j}=\left(\sigma\left(u_{x}^{j}\right)+u_{x t}^{j}\right)_{x}, \quad 0<x<1, t>t_{j} \\
u^{j}(0, t)=0, \quad\left(\sigma\left(u_{x}^{j}\right)+u_{x t}^{j}\right)(1, t)=P_{j} \quad \text { for } t>t_{j} \\
u_{x}^{j}\left(x, t_{j}\right)=u_{x}^{j-1}\left(x, t_{j}\right)+u_{0}^{j}(x) \quad \text { for } 0 \leqq x \leqq 1 . \\
u_{t}^{j}\left(x, t_{j}\right)=u_{t}^{j-1}\left(x, t_{j}\right)+u_{1}^{j}(x)
\end{gathered}
$$


(For $j=0$ in (7.3), take $u_{x}^{-1}=0=u_{t}^{-1}$ ). The displacement of the bar under the influence of the varying loads and imposed fluctuations is represented by the function

$$
u(x, t)=u^{j}(x, t) \quad \text { if } t_{j} \leqq t<t_{j+1} .
$$

As a "measurement" of the bar, we will consider the sequence of asymptotic states determined by $u^{j}(x, t)$ : Theorem 6.1 will guarantee that there exist $w_{\infty}^{j} \in$ $L^{\infty}(0,1)$ so that

$$
u_{x}^{j}(x, t) \rightarrow w_{\infty}^{j}(x) \quad \text { as } t \rightarrow \infty \quad \text { for a.e. } x \text { in }(0,1) .
$$

The "asymptotic length" of the bar while $t_{j}<t<t_{j+1}$ may then be defined by

$$
L^{j}=\int_{0}^{1} w_{\infty}^{j}(x) d x
$$

and may be considered to correspond to the quantity typically measured in experiment.

Hysteresis. Let us illustrate how hysteresis can occur in this model upon slowly raising, then lowering, the load. Suppose the initial load is small, $P_{0}<P_{\beta}$. There is a unique equilibrium state at this load, for which the strain $u_{x}$ is constant, so $w_{\infty}^{0}(x)=L^{0}=w_{\alpha}\left(P_{0}\right)$. Roughly speaking, we shall show that if the load is raised slowly, and the fluctuations are small, then the measured deformations $L^{j}$ increase along the curve $w_{\alpha}(P)$ until $P$ is close to $P_{\alpha}$, then jump to the curve $w_{\beta}(P)$ when the load $P$ exceeds $P_{\alpha}$. If then the load is decreased slowly, the observed deformations decrease along the curve $w_{\beta}(P)$ until $P$ is close to $P_{\beta}$, then jump back to the curve $w_{\alpha}(P)$ when $P$ drops below $P_{\beta}$.

We proceed with more precision: Given any $P_{f}<P_{\alpha}$, we will show that if the stress increments $P_{j+1}-P_{j}$ are small (see (7.10)), the fluctuations $\left(u_{0}^{j}, u_{1}^{j}\right)$ are small (see (7.11), (7.12)) and the time increments $t_{j+1}-t_{j}$ large (depending on the solutions $u^{j}(x, t)$ themselves), then the sequence of asymptotic states will be a sequence of constant states $w_{\infty}^{j}(x) \equiv L^{j}=w_{\alpha}\left(P_{j}\right)$ which in finitely many steps $N$ can achieve $P_{N}=P_{f}$ with $w_{\infty}^{N}(x) \equiv L^{N}=w_{\alpha}\left(P_{f}\right)$. Thus, by "proceeding slowly and carefully enough," the asymptotic state can be put as close as desired to $w_{\alpha}\left(P_{\alpha}\right)=\alpha$.

Precise conditions on the load increments and fluctuations are as follows: To guarantee global existence of the $u^{j}(x, t)$ and ensure that

$$
w_{\infty}^{j}(x) \equiv w_{\alpha}\left(P_{j}\right),
$$

we require ( $c f$. Theorem 6.1)

$$
\begin{gathered}
\int_{0}^{1}\left(\frac{1}{2} u_{t}^{j^{2}}+W\left(u_{x}^{j}\right)-P_{j} u_{x}^{j}\right)\left(y, t_{j}\right) d y<\frac{1}{2} E_{\alpha}\left(P_{j}\right)^{2}+W\left(w_{\alpha}\left(P_{j}\right)\right)-P_{j} w_{\alpha}\left(P_{j}\right) \\
\left|\int_{1}^{x} u_{t}^{j}\left(y, t_{j}\right) d y\right|<E_{\alpha}\left(P_{j}\right) \quad 0<x<1
\end{gathered}
$$


and

$$
q^{j}\left(x, t_{j}\right)=u_{x}^{j}\left(x, t_{j}\right)+\int_{1}^{x} u_{t}^{j}\left(y, t_{j}\right) d y \in B\left(w_{\alpha}\left(P_{j}\right) ; E_{\alpha}\left(P_{j}\right)\right) \quad \text { for a.e. } x \text { in }(0,1) .
$$

If $P_{j}$ is to be chosen less than $P_{\beta}$, so that $E_{\alpha}\left(P_{j}\right)=\infty$, then no restriction need be imposed on the fluctuation $\left(u_{0}^{j}, u_{1}^{j}\right)$ or the time $t_{j}>t_{j-1}$ to ensure (7.4). If $P_{j}>P_{\beta}$, though, $E_{\alpha}\left(P_{j}\right)$ will be finite. We may assume (as an induction hypothesis) that $w_{\infty}^{j-1}(x) \equiv w_{\alpha}\left(P_{j-1}\right)$ and

$$
\begin{gathered}
\int_{0}^{1}\left(\frac{1}{2} u_{t}^{j-1^{2}}+W\left(u_{x}^{j-1}\right)-P_{j-1} u_{x}^{j-1}\right)(y, t) d y=R_{j-1}(t)+W\left(w_{\alpha}\left(P_{j-1}\right)\right) \\
-P_{j-1} w_{\alpha}\left(P_{j-1}\right) \quad \text { where } R_{j-1}(t) \rightarrow 0 \quad \text { as } t \rightarrow \infty .
\end{gathered}
$$

From the initial conditions (7.3) for $u^{j}$, the left side of (7.5) may be expressed as

$$
\begin{gathered}
\left\{R_{j-1}\left(t_{j}\right)-\left(P_{j}-P_{j-1}\right) \int_{0}^{1}\left(u_{x}^{j-1}\left(y, t_{j}\right)-w_{\alpha}\left(P_{j-1}\right)\right) d y\right\} \\
(7.9)+\left\{\int_{0}^{1}\left[u_{t}^{j-1}\left(t_{j}\right) u_{1}^{j}+\frac{1}{2} u_{1}^{j}+W\left(u_{x}^{j-1}\left(t_{j}\right)+u_{0}^{j}\right)-W\left(u_{x}^{j-1}\left(t_{j}\right)\right)-P_{j} u_{0}^{j}\right](y) d y\right\} \\
+\left\{W\left(w_{\alpha}\left(P_{j-1}\right)\right)-P_{j} w_{\alpha}\left(P_{j-1}\right)\right\} .
\end{gathered}
$$

Require that $P_{j}$ satisfy

$$
\left|W\left(w_{\alpha}\left(P_{j-1}\right)\right)-W\left(w_{\alpha}\left(P_{j}\right)\right)-P_{j}\left(w_{\alpha}\left(P_{j-1}\right)-w_{\alpha}\left(P_{j}\right)\right)\right|<\frac{1}{6} E_{\alpha}\left(P_{j}\right)^{2} .
$$

Indeed, the loads $P_{j}$ may be chosen inductively to satisfy (7.10), achieving $P_{N}=P_{f}<P_{\alpha}$ in finitely many steps $N$, since for $P \leqq P_{f}, E_{\alpha}(P) \geqq E_{\alpha}\left(P_{f}\right)>0$. We now need to restrict $t_{j}$ and $\left(u_{0}^{i}, u_{1}^{j}\right)$ so that the first and second brackets in (7.9) are each less than $\frac{1}{6} E_{\alpha}\left(P_{j}\right)^{2}$, and so that (7.6) and (7.7) hold.

For the second bracket in (7.9), it suffices to require

$$
\int_{0}^{1} u_{1}^{j^{2}}+M \int_{0}^{1}\left|u_{0}^{j}\right|<\frac{1}{16} E_{\alpha}^{2}(P)
$$

for some suitably large $M$ depending only on an interval containing all values of strain to be encountered. We require that the fluctuations also satisfy

$$
\left|u_{0}^{j}(x)\right|<\frac{1}{2} E_{\alpha}\left(P_{j}\right) \quad 0 \leqq x \leqq 1 .
$$

Now the first bracket and all the conditions (7.5)-(7.7) hold provided that $t_{j}$ is sufficiently large, depending on the solution $u^{j-1}(x, t)$ at $t=t_{j}$. (Note that the restrictions on the loads $P_{j}$ and the fluctuations $\left(u_{0}^{j}, u_{1}^{j}\right)$ do not depend on the solution, however.)

To summarize, we have shown that if the loads $\boldsymbol{P}_{j}$ satisfy (7.10), the fluctuations $\left(u_{0}^{j}, u_{1}^{j}\right)$ satisfy (7.11), (7.12) and the increments $t_{j}-t_{j-1}$ are sufficiently large, 
then in finitely many steps $N$ we can achieve $w_{\infty}^{N}(x) \equiv w_{\alpha}\left(P_{f}\right)$ for any $P_{f}<P_{\alpha}$ (so $w_{\alpha}\left(P_{f}\right)$ may be as close to as desired to $\alpha$ ).

To conclude our discussion of hysteresis, we note that if the stress is raised more, so that for some $M>N$ we have $P_{M}>P_{\alpha}$, then we must have $w_{\infty}^{M}(x) \equiv w_{\beta}\left(P_{M}\right)$, since this is the unique equilibrium state at stress $P_{M}$. The stress may now be lowered slowly to any level $\tilde{P}_{f}>P_{\beta}$, achieving $w_{\infty}^{L}(x) \equiv w_{\beta}\left(\tilde{P}_{f}\right)$ for some finite $L>M$, provided the fluctuations are restricted, in a manner similar to that above. If now the stress is lowered further to a level less than $P_{\beta}$, of course the asymptotic strain will return to the branch $w_{\alpha}(P)$ (in the $\alpha$ phase), completing a hysteresis loop.

Creep. As $P$ approaches $P_{\alpha}$ from below, the "metastable" state $w_{\alpha}(P)$ becomes more sensitive to perturbations. That is, $E_{\alpha}(P) \rightarrow 0$ and the "basin of stability" of the homogeneous state $w_{\alpha}(P)$ which can be guaranteed by the restrictions (7.11), (7.12) becomes smaller. If fluctuations are larger, nonhomogeneous states may appear, resulting in a measured deformation $L$ which is some average of the available homogeneous states $w_{\alpha}(P)$ and $w_{\beta}(P)$. We will illustrate how a "creep" phenomenon may occur due to small-energy fluctuations of sensitive metastable states producing nonhomogeneous asymptotic states. (Such small-energy fiuctuations might be considered a primitive model for the effect of Brownian motion in the bar.)

Consider the experiment (7.1)-(7.3) carried out at a constant load $P<P_{\alpha}$, so $P_{j}=P$ for all $j$. Suppose that $P$ is close to $P_{\alpha}$, so that

$$
E=E_{\alpha}(P) \ll E_{\beta}(P) .
$$

So that we may apply Theorem 6.1 (by verifying (6.3), (6.4)) to deduce the invariance of the interval $B\left(w_{a}(P) ; E\right)$ for the modified strain $q$, we need two hypotheses: First, we require that the fluctuations have small energy in the sense that (7.11) is satisfied for some suitably large $M$, which now may depend on how small $E$ is. Second, we assume that each asymptotic state $w_{\infty}^{j}(x)$ takes the "unstable" value $w_{u}$ (where $w_{u}$ is the middle solution of $\sigma(w)=P, w_{\alpha}(P)<w_{u}<w_{\beta}$ ) on a set of negligible measure. (This assumption is physically reasonable in the sense that an equilibrium state $\tilde{w}(x)$ having $\tilde{w}(x)=w_{u}$ on a set of positive measure is unstable, by 4.2 .)

To verify that these hypotheses suffice to make (6.3) hold in the form

$$
\int_{0}^{1}\left(\frac{1}{2} u_{t}^{j^{2}}+W\left(u_{x}^{j}\right)-P v_{x}^{j}\right)\left(y, t_{j}\right) d y<\frac{1}{2} E_{\alpha}(P)^{2}+\int_{0}^{1} W_{m}\left(q^{j}\left(y, t_{j}\right)\right) d y,
$$

set

$$
W_{P}(w)=W(w)-P w
$$

observe that

$$
\int_{0}^{1} W_{P}\left(u_{x}^{j-1}\right)(t)=\int_{0}^{1} W_{P}\left(w_{\infty}^{j-1}\right)+\tilde{R}_{j-1}(t)
$$


where $\tilde{R}_{j-1}(t) \rightarrow 0$ as $t \rightarrow \infty$, and write

$$
\begin{aligned}
\int_{0}^{1} W_{P}\left(u_{x}^{j-1}\left(t_{j}\right)+u_{0}^{j}\right)-W_{m}\left(q^{j}\left(t_{j}\right)\right) \\
=\int_{0}^{1}\left(W_{P}\left(u_{x}^{j-1}\left(t_{j}\right)+u_{0}^{j}\right)-W_{P}\left(u_{x}^{j-1}\left(t_{j}\right)\right)\right)+\int_{0}^{1}\left(W_{P}\left(w_{\infty}^{j-1}\right)-W_{m}\left(w_{\infty}^{j-1}\right)\right) \\
\quad+\int_{0}^{1}\left(W_{m}\left(w_{\infty}^{j-1}\right)-W_{m}\left(q^{j}\left(t_{j}\right)\right)\right)+\tilde{R}_{j-1}\left(t_{j}\right)=T_{1}+T_{2}+T_{3}+\tilde{R}_{j-1}\left(t_{j}\right) .
\end{aligned}
$$

Now estimate

$$
\begin{gathered}
\left|T_{1}\right|<M_{1} \int_{0}^{1}\left|u_{0}^{j}\right| \\
\left|T_{2}\right|<M_{2} \mu\left\{x \mid w_{\infty}^{j-1}(x)=w_{u}\right\} \\
\left|T_{3}\right|<M_{3}\left(\mu\left(S_{1}\right)+\mu\left(S_{2} \cap S_{3}\right)\right)
\end{gathered}
$$

where

$$
\begin{gathered}
S_{1}=\left\{x \mid w_{\infty}^{j-1}(x)=w_{\alpha}(P) \quad \text { and } \quad\left|u_{x}^{j-1}\left(x, t_{j}\right)-w_{\infty}^{j-1}(x)\right|>E / 4\right\}, \\
S_{2}=\left\{x \mid w_{\infty}^{j-1}(x)=w_{\alpha}(P) \quad \text { and } \quad\left|u_{x}^{j-1}(x)-w_{\infty}^{j-1}(x)\right|<E / 4\right\}, \\
S_{3}=\left\{x|| u_{0}^{j}(x) \mid>E / 4\right\} .
\end{gathered}
$$

(Provided that $\int u_{t}^{j-1}\left(t_{j}\right)^{2}<E^{2} / 16,\left|q^{j}\left(x, t_{j}\right)-w_{\alpha}(P)\right|>E$ and $x \in S_{2}$ imply $x \in S_{3}$.) Now $\mu\left(S_{1}\right) \rightarrow 0$ as $t_{j} \rightarrow \infty, \mu\left(S_{2}\right)$ is negligible by assumption, and $\mu\left(S_{3}\right) \leqq \frac{4}{E} \int_{0}^{1}\left|u_{0}^{j}\right|$. Thus if $t_{j}$ is large, (7.13) holds.

The third hypothesis we impose on the experiment (7.1)-(7.3) in the present discussion of creep is to require the fluctuations in strain to satisfy

$$
\left|u_{0}^{j}(x)\right| \leqq M_{0}, \quad \text { where } 5 E<M_{0}<2 E_{\beta}(P)-4 E .
$$

Thus the restriction (7.12) is considerably relaxed, since we may take $M_{0} \gg E$. Roughly speaking, the restriction (7.14) will imply that fluctuations cannot "kick" the strain from the $\beta$ phase to the $\alpha$ phase, but that they can "kick" the strain at some points into the $\beta$ phase from the $\alpha$ phase (but only on a small set compatible with (7.11)). Given suitable perturbations (see (7.16)), the result is that the set of points $x$ such that $w_{\infty}^{j}(x)=w_{\beta}(P)$ grows, and the set of $x$ with $w_{\infty}^{j}(x)=$ $w_{\alpha}(P)$ gets smaller, so that the asymptotic length $L^{j}$ slowly grows. This is the creep phenomenon desired.

To be more precise, set

$$
S_{\beta}^{j-1}=\left\{x|| q^{j-1}\left(x, t_{j}\right)-w_{\beta}(P) \mid<2 E\right\}
$$

and observe

$$
\left\{x \mid w_{\infty}^{j-1}(x)=w_{\alpha}(P)\right\} \subset\left(S_{\beta}^{j-1}\right)^{c}=[0,1] \backslash S_{\beta}^{j-1} .
$$

We will show that if $t_{j+1}$ is large enough, then

$$
S_{\beta}^{j} \supseteq S_{\beta}^{j-1} \cup\left\{x \mid u_{0}^{j}>5 E\right\} \text {. }
$$


It follows that if the fluctuations satisfy, for some $\varepsilon>0$,

$$
\mu\left(\left(S_{\beta}^{j-1}\right)^{c} \cap\left\{x \mid u_{0}^{j-1}>5 E\right\}\right) \geqq \varepsilon \mu\left(\left(S_{\beta}^{j-1}\right)^{c}\right),
$$

then $\mu\left(\left(S_{\beta}^{j}\right)^{c}\right) \leqq \mu\left(\left(S_{\beta}^{j-1}\right)^{c}\right)(1-\varepsilon)$, so that

$$
\mu\left(\left(S_{\beta}^{j}\right)^{c}\right) \leqq(1-\varepsilon)^{j}
$$

which implies "creep".

It remains to establish (7.15). We assume $t_{j}$ is so large that $q^{j-1}\left(x, t_{j}\right)>$ $w_{\alpha}(P)-E$ for all $x \in[0,1]$. Then, because of the condition on $M_{0}$ in (7.14), and (7.11), we find that if $u_{0}^{i}(x)>5 E$, then $q^{j}\left(x, t_{j}\right)>w_{\beta}(P)-2 E_{\beta}(P)+2 E$. Since $\sigma(w)-P$ is strictly negative on $\left(w_{\beta}(P)-2 E_{\beta}(P)+E, w_{\beta}(P)-E\right)$ and strictly positive on $\left(w_{\beta}(P)+E, \infty\right)$, we can ensure that for $t_{j+1}-t_{j}$ larger than some constant, we have $\left|q^{j}\left(x, t_{j+1}\right)-w_{\beta}(P)\right|<2 E$, that is, $x \in S_{\beta}^{j}$. This establishes (7.15), and concludes our discussion of creep.

\section{Admissibility and Moving Phase Boundaries in an Elastic Bar}

\section{A Viscosity Criterion for Admissibility of Waves}

If one deals with the elastic system (2.2), one must deal with discontinuous weak solutions. Here the ill-posedness of (2.2) is evident in the simplest initialvalue problem involving discontinuous data, the Riemann problem: One seeks a centered wave solution $(w, v)(x / t)$ of $(2.2)$ with initial data

$$
(w, v)(x, 0)=\left\{\begin{array}{ll}
\left(w_{-}, v_{-}\right) & \text {for } x<0 \\
\left(w_{+}, v_{+}\right) & \text {for } x>0
\end{array} .\right.
$$

Then with $\sigma(w)$ of the form in Fig. 1, JAMES (1980 b) has shown that the Riemann problem can have a two-parameter family of centered wave weak solutions, so uniqueness fails in a rather bad way.

To resolve this issue of uniqueness seems to require selecting of those discontinuous waves for (2.2) of the form

$$
(w, v)(x, t)= \begin{cases}\left(w_{-}, v_{-}\right) & \text {for } x<s t \\ \left(w_{+}, v_{+}\right) & \text {for } x>s t\end{cases}
$$

that are to be regarded as physically relevant, or admissible. The approach we will follow is to identify those waves which arise in the limit of vanishing viscosity.

For the wave (8.1) to be a weak solution of (2.2), the Rankine-Hugoniot jump conditions must hold:

$$
\begin{gathered}
-s\left(w_{+}-w_{-}\right)=\left(v_{+}-v_{-}\right), \\
-s\left(v_{+}-v_{-}\right)=\sigma\left(w_{+}\right)-\sigma\left(w_{-}\right) .
\end{gathered}
$$

The second equation may be replaced by

$$
s^{2}\left(w_{+}-w_{-}\right)=\sigma\left(w_{+}\right)-\sigma\left(w_{-}\right) .
$$


We will identify such a wave as admissible according to the viscosity criterion provided that

$$
(w, v)(x, t)=\lim _{\mu \rightarrow 0^{+}}\left(w^{\mu}, v^{\mu}\right)(x, t)
$$

where $\left(w^{u}, v^{\mu}\right)(x, t)$ are traveling wave solutions of the associated viscoelastic system derived from (1.1):

$$
\begin{gathered}
w_{t}=v_{x} \\
v_{t}=\left(\sigma(w)+\mu w_{t}\right)_{x} .
\end{gathered}
$$

The traveling wave solutions may be taken in the form $(\phi, \psi)((x-s t) / \mu)$ where $\phi, \psi$ satisfy

$$
\begin{gathered}
\lim _{\xi \rightarrow \pm \infty}(\phi, \psi)(\xi)=\left(w_{ \pm}, v_{ \pm}\right), \\
-s\left(\phi-w_{-}\right)=\psi-v_{-}, \\
-s\left(\psi-v_{-}\right)=\sigma(\phi)-\sigma\left(w_{-}\right)-s \phi^{\prime} .
\end{gathered}
$$

The last equation may be replaced by

$$
s \phi^{\prime}(\xi)=\sigma(\phi)-\sigma\left(w_{-}\right)-s^{2}\left(\phi-w_{-}\right) .
$$

There are now two cases. If $s \neq 0$ and $\left(w_{+}, w_{-}, s\right)$ satisfies (8.2), then (8.5) is an ordinary differential equation, and it is well known and easily verified that a solution exists satisfying $\lim _{\xi \rightarrow \pm \infty} \phi(\xi)=w_{ \pm}$if and only if the following chord condition is satisfied:

(8.6) The chord connecting the points $\left(w_{ \pm}, \sigma\left(w_{ \pm}\right)\right)$should lie above (respectively below) the graph of $\sigma(w)$ if ( $\operatorname{sgn} s) \operatorname{sgn}\left(w_{+}-w_{-}\right)$is negative (respectively positive).

This condition ensures that $\operatorname{sgn} \phi^{\prime}=\operatorname{sgn}\left(w_{+}-w_{-}\right)$when $\phi$ is between $w^{+}$and $w^{-}$, and would be equivalent to LIU's (strict) entropy condition for shocks (LIU, 1976) if the system were strictly hyperbolic (which requies $\sigma^{\prime}>0$ everywhere).

In the second case, $s=0$, so (8.5) reduces to an algebraic equation

$$
\sigma(\phi)=\sigma\left(w_{-}\right) \text {. }
$$

A solution with the desired property is

$$
\phi(\xi)=w(\xi)= \begin{cases}w_{-} & \text {for } \xi<0 \\ w_{+} & \text {for } \xi>0\end{cases}
$$

As we have already noted, the viscoelastic equation (1.1) has the same equilibria as the elastic equation (2.1). Thus all elastic equilibria are trivial viscous limits.

To summarize, the viscosity criterion for admissibility of weak solutions of (2.2) of the form (8.1) may be stated as follows:

(8.9) If $s \neq 0$, the wave is admissible if the chord condition (8.6) holds. If $s=0$, the wave is admissible unconditionally. 


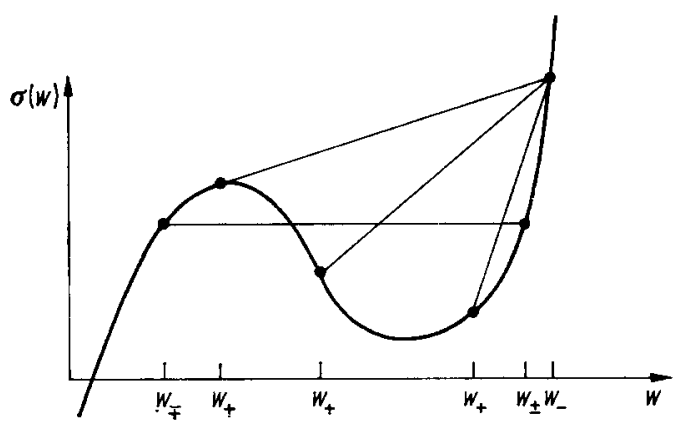

Fig. 6. Pairs $\left(w_{+}, w_{-}\right)$determining admissible waves for $s \geq 0$

Concerning the solution of the Riemann problem under the admissibility criterion (8.9), it is remarkable that a study already exists. For $\sigma(w)$ as in Fig. 1, changing convexity at just one point, SHEARER (1982) established existence and uniqueness of the solution, provided that discontinuities are required to satisfy exactly the admissibility condition (8.9). SHEARER appears to have admitted the waves with $s=0$ on an ad hoc basis. Here we have shown how these waves arise from the viscosity criterion.

SLEMROD (1983) has previously dismissed a viscosity criterion of the sort above, his analysis forbidding the waves having $s=0$. The waves remaining form a class insufficient to solve the Rjemann problem. However, SLEMROD rules out a priori discontinuous viscous waves of the sort in (8.8). In the hindsight afforded by the results of $\S 6$ above, his requirement of continuity seems clearly too restrictive. Discontinuous stationary waves of this sort can be stable asymptotic limits of smooth solutions in the viscoelastic problem (2.4).

SHEARER (1983) and also SLEMROD (1983) have examined admissibility criteria based on other forms of dissipation mechanisms in the system (8.3). SLEMROD's viscosity-capillarity criterion admits just a single stationary wave, at the Maxwell line, where the stored-energy density at $w_{+}$and $w_{-}$are equal. (Also see AifanTIS \& SERrIN (1983).) Typically, for moving waves, SLEMrod's criterion implies that the metastable state is overtaken by the stable state. A recent result of SHEARER (1986) indicates, however, that the viscosity-capillarity criterion may fail to imply uniqueness of solutions of some Riemann problems with states near the Maxwell line.

We finish with two remarks concerning the admissible waves selected by the viscosity criterion developed in this section. We fix $\sigma$ of the form in Figure 1, requiring that the convexity of $\sigma$ changes at just one point.

We first remark that the closure of the set triples $\left(w_{\dashv}, w_{-}, s\right)$ which determine an admissible wave (2.2) form a connected continuum in $\mathbb{R}^{3}$. That is, any two triples $\left(w_{+}, w_{-}, s\right)$ and $\left(\tilde{w}_{+}, \tilde{w}_{-}, \tilde{s}\right)$ which determine admissible waves can be connected by a path in this set. We leave the verification to the reader. Note that this includes waves with speed of any sign, so the zero speed "shocks" in particular are not exceptional from this point of view.

We shall have occasion in $\$ 9$ to consider "interphase shocks," for which $w_{+}$and $w_{-}$lie in different components of $\left\{w \mid \sigma^{\prime}(w) \geqq 0\right\}$. Waves of this sort exist 
with arbitrarily small speed $s$. Consider the "shock structure" for these wave obtained from (8.5). The second remark is that if viscosity $\mu>0$ is fixed, the smooth "transition layer" obtained from (8.5) for interphase shocks approaches zero thickness as $s \rightarrow 0$. For $s>0, w_{+}<\alpha$, and $w_{-}>\beta$ in Fig. 1, the condition $s \rightarrow 0$ implies $w_{+} \rightarrow \alpha$ and $w_{-} \rightarrow \gamma$, where $\gamma=w_{\beta}(\sigma(\alpha))$ in the notation of $\S 7$. Our second remark means that, fixing the phase of the wave appropriately, as $s \rightarrow 0$ we have

$$
\phi(\xi) \rightarrow \phi_{0}(\xi)= \begin{cases}\gamma & \text { for } \xi<0 \\ \alpha & \text { for } \xi>0\end{cases}
$$

\section{Appearance of Propagating Phase Boundaries}

In the hysteresis process discussed in $\S 7$, a phase transition occurs approximately when the load level $P$ crosses the level $P_{\alpha}$. The bar, formerly in the " $\alpha$ phase" with asymptotic strain $w_{\alpha}(P)$, is forced into the " $\beta$ phase" with asymptotic strain $w_{\beta}(P)$. We describe here an idealized problem for the elastic bar indicating that this transition can be associated with a slowly propagating "phase boundary", corresponding to a wave of discontinuous strain for the system (2.2) which is admissible according to the chord condition (8.6). The mechanism I suggest has been examined by PENCE (1986) and suggested in the context of shear flow in polymeric fluids by HuNTER \& SLEMROD (1983). However, we are able to assert the uniqueness of the solution to the problem (9.1) below within the class of centered wave solutions satisfying the admissibility criterion (8.9) derived from the limit of vanishing viscosity.

For ease in applying the results of SHEARER (1982), we will in fact consider the situation in which the load $P$ falls the below transition level $P_{\beta}$ (notation as in $\S 7$ ), with the bar previously equilibrated in the $\beta$ phase.

Suppose then that at $t=0$ the elastic bar lies at equilibrium at a stress level $P>P_{\beta}, P$ near $P_{\beta}$, so $w(x, 0) \equiv w_{\beta}(P)>\beta, v(x, 0) \equiv 0$ in the system (2.2), for $0 \leqq x \leqq 1$. Imagine that the load at the boundary $x=1$ is suddenly lowered to a level $P_{+}<P_{\beta}$, and held constant. In the elastic model, this determines the strain at the boundary to be $w(1, t)=w_{\alpha}\left(P_{+}\right)<\alpha$, since we must have $\sigma(w(1, t))=P_{+}$.

We now have the following initial-boundary value problem (a Riemann problem at the boundary):

(9.1) Find a weak solution of the elastic system (2.2) having initial conditions

$$
w(x, 0)=w_{\beta}(P), \quad v(x, 0)=0 \quad 0 \leqq x \leqq 1
$$

and boundary condition

$$
w(1, t)=w_{\alpha}\left(P_{+}\right) \quad 0<t .
$$

(For short time, we ignore the fixed-end condition $v(0, t)=0$.) We can construct a solution to this problem for short time by finding a centered 1-wave solution to the ordinary Riemann problem for $(2.2)$, centered at $(x, t)=(1,0)$, connecting 
$\left(w_{-}, v_{-}\right)=\left(w_{\beta}(P), 0\right)$ on the left to $\left(w_{+}, v_{+}\right)=\left(w_{\alpha}(P), v_{+}\right)$on the right, for some $v_{+}$to be determined. That is, we seek $v_{+}$such that the solution of the Riemann problem for $\left(w_{-}, v_{-}\right),\left(w_{+}, v_{+}\right)$contains only 1 -waves, which have negative speed, so that the boundary condition in (9.1) will be satisfied. The resulting solution is valid until the leading wave impinges on the boundary $x=0$.

The problem in (9.1) is now easily resolved utilizing SHEARER's solution (SHEARER, 1982) of the Riemann problem for (2.2) under the admissibility criterion justified in $\S 8$. We must assume $\sigma^{\prime \prime}(w)<0$ for $w<\alpha, \sigma^{\prime \prime}(w)>0$ for $w>\beta$. We find that a unique $v_{+}=v_{b}<0$ with the property described above does always exist. In SHEARER's notation, given the point $U_{0}=\left(w_{-}, v_{-}\right)$in his Figure 4, we require that the solution involve only 1 -waves, so that the state $U_{1}=\left(w_{+}, v_{+}\right)$ must lie either on the curve $S_{1}^{*}\left(U_{0}\right)$ or on the curve $E$. The structure of the solution is as follows: If $w_{+} \leqq w_{*}\left(w_{-}\right)$where the tangent to the graph of $\sigma(w)$ at $w=w_{-}$ intersects the graph again at $w_{*}\left(w_{-}\right)<\alpha$, then the solution is a single 1-shock connecting $\left(w_{-}, v_{-}\right)$to $\left(w_{+}, v_{+}\right)$. If $w_{*}\left(w_{-}\right)<w_{+}<w_{\alpha}\left(P_{\beta}\right)$, then the solution consists of a rarefaction wave connecting $\left(w_{-}, v_{-}\right)$to an intermediate state $\left(w_{0}, v_{0}\right)$, with $\beta<w_{0}<w_{-}$, which is then immediately connected to $\left(w_{+}, v_{+}\right)$by a 1 shock. Here $w_{0}$ is determined by the requirement $w_{*}\left(w_{0}\right)=w_{+}$, that is, the chord from $\left(w_{+}, \sigma\left(w_{+}\right)\right)$to $\left(w_{0}, \sigma\left(w_{0}\right)\right)$ lies below the graph of $\sigma(w)$ and is tangent to it at $w_{0}$. As $P_{+} \uparrow P_{\beta}$, or as $w_{+}$approaches $w_{\alpha}\left(P_{\beta}\right)$, clearly the slope of this chord, which is equal to the squared speed of the shock connecting the two phases, approaches zero. Thus the interphase shock which appears in the solution can have an arbitrarily small speed, depending on how close $P_{+}$is to $P_{\beta}$. We also note that as $P_{+} \rightarrow P_{\beta}$, the velocity $v_{b} \rightarrow 0$.

We conclude by illustrating a "necking" phenomenon arising out of a Riemann problem for (2.2) obtained by applying a symmetry principle to the solutions just described. (Actually, true "necking" would arise in the companion situation for loads near $P_{\alpha}$.) Denote the solution of the whole-line Riemann problem just used to solve $(9.1)$, centered at $(x, t)=(0,0)$, by $(\tilde{w}, \tilde{v})(x, t)$, so that

$$
(\tilde{w}, \tilde{v})(x, 0)=\left\{\begin{array}{ll}
\left(w_{\beta}(P), 0\right) & \text { for } x<0 \\
\left(w_{\alpha}\left(P^{+}\right), v_{b}\right) & \text { for } x>0
\end{array}\right. \text {. }
$$

Then for $t \geqq 0$ and all $x \geqq 0$, we know $(\tilde{w}, \tilde{v})(x, t)=\left(w_{\alpha}\left(P_{+}\right), v_{b}\right)$ as well. Due to the symmetry of the equations (2.2) and the chord condition (8.6), the function

$$
(w, v)(x, t)= \begin{cases}(\tilde{w}, \tilde{v})(x, t) & \text { for } x<0 \\ \left(\tilde{w}, 2 v_{b}-\tilde{v}\right)(-x, t) & \text { for } x>0\end{cases}
$$

is the (unique admissible) solution of the Riemann problem for (2.2) with initial states

$$
\left(w_{-}, v_{-}\right)=\left(w_{\beta}(P), 0\right), \quad\left(w_{+}, v_{+}\right)=\left(w_{\beta}(P), 2 v_{b}\right) .
$$

Physically, this means starting with homogeneous strain $w=w_{\beta}(P)$ at $t=0$, but giving the right "half" of the bar a small velocity $2 v_{b}<0$. The solution then develops a slowly expanding region near $x=0$ in the $\alpha$ phase with $w=w_{\alpha}\left(P_{+}\right)$. 
Acknowledgements. I have benefitted from very useful discussions with JERRY ERICKsen, John Ball, Hans Weinberger, Fred Weissler and Fred Hickernell. This work was largely performed while on leave at the Institute for Mathematics and its Applications at the University of Minnesota. I also acknowledge the support of the Mathematics Research Center at the University of Wisconsin, where I performed numerical experiments which led to this work. This work was partially supported by the National Science Foundation under Grant DMS-84-01614.

\section{Appendix: Some Estimates for the Abstract Parabolic Equation}

Essentially, in this section we reorganize the proof of HENRY (1981), 3.5.2 to obtain estimates needed in $\S 3$ and $\S 5$. Throughout, we assume $A$ is sectorial on a Banach space $X, f: U \rightarrow X$ is locally Lipschitz-continuous on an open set $U \subset \mathbb{R} \times X^{\alpha}$ for some $0 \leqq \alpha<1$, and $z(t)$ is a solution on $(0, T]$ of

$$
z_{t}+A z=f(t, z), \quad z(0)=z_{0}
$$

with $\left(0, z_{0}\right) \in U$. We assume

$$
\|f(t, z(t))-f(s, z(s))\| \leqq K(t-s)+L\|z(t)-z(s)\|_{\alpha} .
$$

(Here \|\| is the norm in $X,\|\|_{\alpha}$ the norm in $X^{\alpha}$.) We let

$$
M_{z}=\left\|z_{0}\right\|_{x} \quad M_{f}=\sup _{0 \leqq t \leqq T}\|f(t, z(t))\| .
$$

Below, we rely heavily on standard estimates for fractional powers of sectorial operators (HENRY 1.4.3).

Lemma A.1. If $\alpha \leqq \beta<1$, there exists $C=C(\alpha, \beta, T)$ so that

$$
\|z(t)\|_{\beta} \leqq C\left(t^{\alpha-\beta} M_{z}+M_{f}\right) \quad \text { for } 0<t<T .
$$

Proof. The solution $z(t)$ satisfies

$$
z(t)=e^{-A t} z_{0}+\int_{0}^{t} e^{-A(t-s)} f(s, z(s)) d x .
$$

Using standard estimates (HENRY 1.4.3), we find

$$
\|z(t)\|_{\beta} \leqq C M_{z} t^{\alpha-\beta}+C M_{f} \int_{0}^{t} s^{-\beta} d s .
$$

The lemma follows.

Lemma A.2. There exists $C_{*}=C_{*}(\alpha, T, L)$ such that for $0<t<t+h<T$ we have

$$
\|z(t+h)-z(t)\|_{\alpha} \leqq h t^{-1} C_{*}\left(M_{z}+M_{f}+K\right)
$$


Proof. Fix $0<\tau<1$ and write, for $t>\tau$,

$$
\begin{gathered}
z(t+h)=e^{-A(t+h-\tau)} z(\tau)+\left(\int_{\tau}^{\tau+h}+\int_{\tau+h}^{t+h}\right) e^{-A(t+h-s)} f(s, z(s)) d s, \\
z(t+h)-z(t)=\left(e^{-A h}-I\right) e^{-A(t-\tau)} z(\tau)+\int_{\tau}^{\tau+h} e^{-A(t+h-s)} f(s, z(s)) d s \\
+\int_{\tau}^{t} e^{-A(t-s)}(f(s+h, z(s+h))-f(s, z(s))) d s=T_{1}+T_{2}+T_{3} .
\end{gathered}
$$

Now

$$
\begin{aligned}
\left\|T_{3}\right\|_{\alpha} & \leqq C \int_{\tau}^{t}(t-s)^{-\alpha}\left(K h+\|z(s+h)-z(s)\|_{\alpha}\right) d s \\
& \leqq C K h+C \int_{\tau}^{t}(t-s)^{-\alpha}\|z(s+h)-z(s)\|_{\alpha} d s \\
\left\|T_{2}\right\|_{\alpha} & \leqq C M_{f} \int_{\tau}^{\tau+h}(t+h-s)^{-\alpha} d s \leqq h C M_{f}(t-\tau)^{-\alpha} .
\end{aligned}
$$

Choosing $\beta$ with $\alpha<\beta<1$, and again using estimates of Henry 1.4.3, we have

Now we have

$$
\left\|T_{1}\right\|_{\alpha} \leqq h C(t-\tau)^{\beta-\alpha-1}\|z(\tau)\|_{\beta} .
$$

$$
\begin{aligned}
\|z(t+h)-z(t)\|_{\alpha} h^{-1} \leqq & (t-\tau)^{\beta-\alpha-1} C\left(\|z(\tau)\|_{\beta}+M_{f}+K\right) \\
& +C L \int_{\tau}^{t}(t-s)^{-\alpha}\|z(s+h)-z(s)\|_{\alpha} h^{-1} d s .
\end{aligned}
$$

Because $\beta-\alpha-1>-1$ and $0 \leqq \alpha<1$ we may now apply the generalized Gronwall inequality (HeNRY (1981), Ch. 6, ex. 4) to conclude that

$$
\|z(t+h)-z(t)\|_{\alpha} \leqq h C_{*}(t-\tau)^{\beta-\alpha-1}\left(\|z(\tau)\|_{\beta}+M_{f}+K\right)
$$

where $C_{*}=C_{*}(\alpha, \beta, T, L, C)$ is independent of $\tau$. Now choose $\tau=t / 2$ and apply Lemma A.1 to obtain the result.

Lemma A.3. If $0<\gamma<1$, there exists $C_{*}=C_{*}(\alpha, \gamma, T, L)$ so that for $0<$ $t<T$,

$$
\left\|z_{t}(t)\right\|_{\gamma} \leqq C_{*}\left(t^{\alpha-\gamma-1} M_{z}+t^{-\gamma}\left(M_{f}+K\right)\right) .
$$

Proof. From the work of HenRy 3.2.1 we may write

$$
\begin{gathered}
z_{t}(t)=-A e^{-A t} z_{0}+e^{-A t} f(t, z(t))+\left(\int_{0}^{t / 2}+\int_{t / 2}^{t}\right) A e^{-A(t-s)}(f(t, z(t)) \\
-f(s, z(s))) d s=T_{1}+T_{2}+T_{3}+T_{4} .
\end{gathered}
$$


We then estimate

$$
\begin{gathered}
\left\|T_{1}\right\|_{\gamma} \leqq C t^{\alpha-\gamma-1} M_{z} \\
\left\|T_{2}\right\|_{\gamma} \leqq C t^{-\gamma} M_{f} \\
\left\|T_{3}\right\|_{\gamma} \leqq C \int_{t / 2}^{t}(t-s)^{-\gamma-1} d s \cdot 2 M_{f} \leqq C t^{-\gamma} M_{f} \\
\left\|T_{4}\right\|_{\gamma} \leqq C \int_{t / 2}^{t}(t-s)^{-\gamma-1}\left(K(t-s)+L\|z(t)-z(s)\|_{\alpha}\right) d s \\
\leqq C_{*} \int_{t / 2}^{t}(t-s)^{-\gamma} s^{-1} d s\left(M_{z}+M_{f}+K\right) \\
=t^{-\gamma} C_{*} \int_{1 / 2}^{1}(1-s)^{-\gamma} s^{-1} d s\left(M_{z}+M_{f}+K\right) .
\end{gathered}
$$

The lemma follows.

\section{References}

E. C. Aifantis \& J. B. Serrin (1983), The mechanical theory of fluid interfaces and Maxwell's rule, J. Coll. Int. Sci. 96, 517-529.

G. ANDREws (1980), On the existence of solutions to the equation $u_{t t}=u_{x x t}+\sigma\left(u_{x}\right)_{x}$, J. Diff. Eqns. 35, 200-231.

G. ANDrews \& J. M. Ball (1982), Asymptotic behaviour and changes of phase in one-dimensional nonlinear viscoelasticity, J. Diff. Eqns. 44, 306-341.

C. M. Dafermos (1969), The mixed initial-boundary value problem for the equations of nonlinear one dimensional viscoelasticity, J. Diff. Eqns. 6, 71-86.

J. L. ERICKSEN (1975), Equilibrium of bars, J. Elasticity 5, 191-201.

J. W. GibBs (1906), On the equilibrium of heterogeneous substances, in The Scientific Papers of J. Willard Gibbs, Longmans, London.

J. M. Greenterg, R. C. MacCamy, \& V. J. Mizel (1968), On the existence, uniqueness and stability of solutions of the equation $\sigma^{\prime}\left(u_{x}\right) u_{x x}+\lambda u_{x x t}=\varrho_{0} u_{t t}$, J. Math. Mech. 17, 707-728.

J. M. GREenderg (1969), On the existence, uniqueness and stability of the equation $\varrho_{0} X_{t t}=E\left(X_{x}\right) X_{x x}+\lambda X_{x x t}$, J. Math. Anal. Appl. 25, 575-591.

J. M. Greenberg \& R. C. MACCAMY (1970), On the exponential stability of solutions of $E\left(u_{x}\right) u_{x x}+\lambda u_{x t x}=\varrho u_{t t}$, J. Math. Anal. Appl. 31, 406-417.

R. HAGAN \& M. SLEMROD (1983), The viscosity-capillarity admissibility criterion for shocks and phase transitions, Arch. Rational Mech. Anal. 83, 333-361.

D. HeNRY (1981), Geometric theory of semilinear parabolic equations, Lecture Notes in Math. v. 840, Springer, New York.

D. Hoff \& J. SMOLLER (1985), Solutions in the large for certain nonlinear parabolic systems, Anal. Non Lin. 2, 213-235.

J. Hunter \& M. Slemrod (1983), Viscoelastic fluid flow exhibiting hysteretic phase changes, Phys. Fluids 26, 2345-2351.

R. D. JAMES (1980a), Coexistent phases in the one dimensional static theory of elastic bars, Arch. Rational Mech. Anal. 72, 99-140. 
R. D. JAmes (1980 b), The propagation of phase boundaries in elastic bars, Arch. Rational Mech. Anal. 73, 125-158.

T.-P. LIU (1976), The entropy condition and the admissibility of shocks, J. Math. Anal. Appl. 53, 78-88.

I. Müller \& K. Wilmanski (1981), Memory alloys-phenomenology and ersatzmodel, in Continuum Models of Discrete Systems 4, eds. O. BRULIN \& R. K. T. HsiEH, NorthHolland, Amsterdam.

T. J. PenCE (1986), On the emergence and propagation of a phase boundary in an elastic bar with a suddenly applied end load. J. Elasticity 16, 3-42.

M. SHEARER (1982), The Riemann problem for a class of conservation laws of mixed type, J. Diff. Eqns. 46, 426-443.

M. SHEARER (1983), Admissibility criteria for shock wave solutions of a system of conservation laws of mixed type, Proc. Roy. Soc. Edinburgh 93 A, 233-244.

M. SHEARER (1986), Nonuniqueness of admissible solutions of Riemann initial value problems for a system of conservation laws of mixed type, Arch. Rational Mech. Anal. 93, 45-59.

M. SLEMrod (1983), Admissibility criteria for propagating phase boundaries in a van der Waals fluid, Arch. Rational Mech. Anal. 81, 301-316.

H. F. WeinBerger (1982), A simple system with a continuum of stable inhomogeneous steady states, Lecture Notes in Num. Appl. Anal. 5, 345-359.

Department of Mathematics The University of Michigan Ann Arbor 